\title{
Complete leading-order standard model corrections to quantum leptogenesis
}

\author{
Paul Frederik Depta, ${ }^{a}$ Andreas Halsch, ${ }^{b}$ Janine Hütig, ${ }^{b}$ Sebastian Mendizabal ${ }^{c}$ and \\ Owe Philipsen ${ }^{b}$
}

a Deutsches Elektronen-Sychrotron DESY, Notkestraße 85, D-22607 Hamburg, Germany

${ }^{b}$ Institut für Theoretische Physik, Goethe-Universität Frankfurt am Main, Max-von-Laue-Str. 1, 60438 Frankfurt am Main, Germany

${ }^{c}$ Department of Physics, Universidad Técnica Federico Santa María, Casilla 110-V, Valparaíso, Chile

E-mail: frederik.depta@desy.de, halsch@itp.uni-frankfurt.de, janine.schaefer1@web.de, sebastian.mendizabal@usm.cl, philipsen@itp.uni-frankfurt.de

ABSTRACT: Thermal leptogenesis, in the framework of the standard model with three additional heavy Majorana neutrinos, provides an attractive scenario to explain the observed baryon asymmetry in the universe. It is based on the out-of-equilibrium decay of Majorana neutrinos in a thermal bath of standard model particles, which in a fully quantum field theoretical formalism is obtained by solving Kadanoff-Baym equations. So far, the leading two-loop contributions from leptons and Higgs particles are included, but not yet gauge corrections. These enter at three-loop level but, in certain kinematical regimes, require a resummation to infinite loop order for a result to leading order in the gauge coupling. In this work, we apply such a resummation to the calculation of the lepton number density. The full result for the simplest "vanilla leptogenesis" scenario is by $\mathcal{O}(1)$ increased compared to that of quantum Boltzmann equations, and for the first time permits an estimate of all theoretical uncertainties. This step completes the quantum theory of leptogenesis and forms the basis for quantitative evaluations, as well as extensions to other scenarios.

Keywords: Resummation, Thermal Field Theory, Beyond Standard Model, Cosmology of Theories beyond the SM

ARXIV EPRINT: 2005.01728 


\section{Contents}

1 Introduction 1

2 Thermal leptogenesis in non-equilibrium quantum field theory 3

2.1 The leptogenesis scenario 3

2.2 Correlation functions and Kadanoff-Baym equations 4

$\begin{array}{ll}2.3 & \text { The lepton number matrix }\end{array}$

3 Gauge corrections to leptogenesis $\quad 7$

3.1 CTL resummation of the Majorana neutrino self-energy 8

$\begin{array}{ll}3.2 & \text { Gauge corrections to lepton self-energies } \\ 3.3 & 12\end{array}$

$\begin{array}{lll}3.3 & \text { Lepton number matrix with complete SM corrections } & 13\end{array}$

4 Evaluation of the lepton number density 13

$\begin{array}{lll}4.1 & \text { The time integrals } & 14\end{array}$

$\begin{array}{ll}4.2 & \text { Approximate integration of the lepton number } \\ \end{array}$

$\begin{array}{lll}4.3 & \text { Time evolution of the lepton number } & 19\end{array}$

4.4 Temperature dependence of the gauge corrections 20

5 Conclusions $\quad 21$

A Feynman diagrams for gauge corrections at three-loop level 22

$\begin{array}{ll}\text { B Leading and sub-leading gauge corrections } & 23\end{array}$

$\begin{array}{ll}\text { C The function } \mathcal{T}\left(t ; \omega_{21}, \omega_{23}, p\right) & 25\end{array}$

D Late-time limit for the lepton number $\quad 25$

$\begin{array}{lll}\text { E SM parameters for the numerical evaluation } & 26\end{array}$

\section{Introduction}

The origin of the observed baryon asymmetry in the universe is an as yet unsolved problem of physics. Although the standard model of particle physics (SM) features baryon plus lepton number $(B+L)$ and $C P$-violating processes before the electroweak phase transition [1], the amount of $C P$ violation is too small to arrive at the observed asymmetry [2]. Moreover, for the experimentally observed Higgs mass of $\sim 125 \mathrm{GeV}$ [3], the electroweak phase transition is merely an analytic crossover [4-6], whereas a sufficiently strong first-order transition is required to provide a departure from equilibrium for baryogenesis. Viable models for 
baryogenesis are thus based on extensions of the SM, with, e.g., additional particles on a GUT scale, which then generate a finite asymmetry via $C P$-violating out-of-equilibrium decays.

A particularly attractive model in this context is thermal leptogenesis [7]. In this case, the SM is extended by three additional right-handed Majorana neutrinos, with Yukawa couplings to the SM Higgs field and left-handed leptons. In the standard scenario [8], these Majorana neutrinos are produced in the early universe at temperatures beyond their mass scale, $T>M$. When the temperature drops below their mass, they decay out-ofequilibrium violating $C P$, such that a lepton asymmetry is generated. Additional washout processes diminish this asymmetry, until they fall out of equilibrium and a finite lepton asymmetry is frozen in. Any lepton asymmetry is partially converted into a baryon asymmetry via SM sphaleron transitions, which violate $B+L$ while keeping $B-L$ constant $[9,10]$. Besides giving robust predictions for baryogenesis, this simple extension of the SM would also explain the smallness of the light neutrino masses via the see-saw mechanism [11]. For recent reviews, also including other scenarios for leptogenesis, see [12-17].

Since leptogenesis is a non-equilibrium process, it is frequently studied using Boltzmann equations, which are inherently classical. For the collision terms, zero temperature matrix elements are used, supplied with thermal momentum distribution functions which introduce quantum effects. However, also off-shell and memory effects become important for non-equilibrium processes and have to be treated appropriately. Further conceptual difficulties arise in gauge theories, where gauge boson numbers are neither gauge invariant nor conserved. The problem of including quantum effects by hand into a classical framework is avoided by a formal, quantum field theoretical treatment based on Kadanoff-Baym equations, which contain everything once computed to sufficient depth in perturbation theory. In [18], quantum corrections to the Majorana neutrino reaction rates due to Higgs and lepton loops have been included, and a detailed comparison with the solutions of Boltzmann equations was given. Qualitative agreement was achieved by additionally introducing thermal damping widths for Higgs and lepton propagators, which are expected to be caused by interactions with the weak gauge bosons and may significantly modify the result at a quantitative level [19].

In this work we complete the quantum mechanical treatment of leptogenesis by calculating gauge corrections systematically. First entering at three-loop level, their evaluation is complicated by the dynamical generation of scales, which necessitate infinite resummations in order to complete even a leading-order result in the gauge coupling. Such resummations have been performed for the Majorana neutrino self-energy and the associated production rate [20]. Here we extend these calculations to the lepton number density in a first complete quantum mechanical calculation of leptogenesis. Our formulation using Kadanoff-Baym equations [18] differs from other field-theoretical approaches, in which rate equations are obtained by exploiting hierarchies of scales in the non-relativistic regime, $T<M$ [21-24].

In order to render this paper self-contained, sections 2 and 3.1 recall the formalism and previous results, which form the basis for our calculation. In section 3.2, we devise a resummation technique to systematically include gauge corrections to the lepton number density, which then is complete to leading order in all SM corrections. In section 4, some 
approximations are motivated which allow for a simple evaluation. Finally, we compare to previous results in the literature and discuss the significance of gauge corrections.

\section{Thermal leptogenesis in non-equilibrium quantum field theory}

\subsection{The leptogenesis scenario}

Starting from the SM, a standard approach to leptogenesis is to add three additional righthanded Majorana neutrinos (which are electroweak singlets) to the Lagrangian $\mathcal{L}_{\mathrm{SM}}$,

$$
\mathcal{L}=\mathcal{L}_{\mathrm{SM}}+\bar{\nu}_{R i} \mathrm{i} \not \partial \nu_{R i}+\bar{l}_{L i} \tilde{\phi} \lambda_{i j}^{*} \nu_{R j}+\bar{\nu}_{R j} \lambda_{i j} l_{L i} \phi-\frac{1}{2} M_{i j}\left(\bar{\nu}_{R i}^{c} \nu_{R j}+\bar{\nu}_{R j} \nu_{R i}^{c}\right)
$$

Here the flavour indices $i, j=1,2,3$ count the families in the gauge eigenbasis, and we denote $\nu_{R}^{c}=C \bar{\nu}_{R}^{T}$, with $C=\mathrm{i} \gamma^{2} \gamma^{0}$ the charge conjugation matrix, and $\tilde{\phi}=\mathrm{i} \sigma_{2} \phi^{*}$. The additional neutrinos are weakly coupled to SM Higgs fields $\phi$ and massless left-handed leptons $l_{L i}$ via Yukawa couplings $\lambda_{i j}$. Without loss of generality, we consider here the simplest scenario, also termed "vanilla leptogenesis", in which the final asymmetry is assumed to be independent of its flavour composition and, in a diagonal mass basis, the Majorana masses $M_{i}$ are hierarchically ordered, $M_{i>1} \gg M_{1}:=M$. For a review including details, viable parameter ranges and other scenarios, see [8]. In this case, the two heavier neutrinos can be integrated out, leading to the effective Lagrangian for the lightest neutrino $N=N_{1}=\nu_{R 1}+\nu_{R 1}^{c}$,

$$
\begin{aligned}
\mathcal{L}= & \mathcal{L}_{\mathrm{SM}}+\frac{1}{2} \bar{N} \mathrm{i} \not \partial N+\bar{l}_{L i} \tilde{\phi} \lambda_{i 1}^{*} N+N^{T} \lambda_{i 1} C l_{L i} \phi-\frac{1}{2} M N^{T} C N \\
& +\frac{1}{2} \eta_{i j} l_{L i}^{T} \phi C l_{L j} \phi+\frac{1}{2} \eta_{i j}^{*} \bar{l}_{L i} \tilde{\phi} C \bar{l}_{L j}^{T} \tilde{\phi}
\end{aligned}
$$

with an effective vertex for lepton and Higgs fields and a combination of Majorana neutrino couplings, respectively,

$$
\eta_{i j}=\sum_{k>1} \lambda_{i k} \frac{1}{M_{k}} \lambda_{k j}^{T}, \quad \lambda^{2}:=\sum_{i}\left|\lambda_{i 1}\right|^{2} .
$$

The heavy neutrino mass is in the range $10^{9} \mathrm{GeV} \lesssim M \lesssim 10^{15} \mathrm{GeV}$ [8], and its coupling is assumed very weak compared to SM couplings $g_{\mathrm{SM}}, \lambda \ll g_{\mathrm{SM}}$. Explicitly, the relevant SM couplings for our work are the $\mathrm{SU}(2)$ and $\mathrm{U}(1)$ gauge group couplings $g$ and $g^{\prime}$, the Yukawa coupling of the top quark $h_{t}$, and the Higgs self coupling $\lambda_{\phi}$, i.e. $g_{\mathrm{SM}} \in\left\{g, g^{\prime}, h_{t}, \sqrt{\lambda_{\phi}}\right\}$.

During the generation of the lepton asymmetry, the heat bath is kept in thermal equilibrium via SM interactions. These act on a time scale $\tau_{\mathrm{SM}} \sim 1 /\left(g_{\mathrm{SM}}^{2} T\right)$, which is much shorter than the equilibration time of the heavy neutrino, $\tau_{N} \sim 1 /\left(\lambda^{2} M\right)$ for $T \gtrsim M$, which thus is out of equilibrium. During this first stage, heavy Majorana neutrinos are produced from zero initial abundance by scattering of leptons and Higgs bosons. This out-of-equilibrium process generates an initial lepton asymmetry, which is later diminished by washout processes. At temperatures $T \sim M$, part of the Majorana neutrinos decay out of equilibrium to produce a final lepton asymmetry, or $B-L>0$, which is of the same 

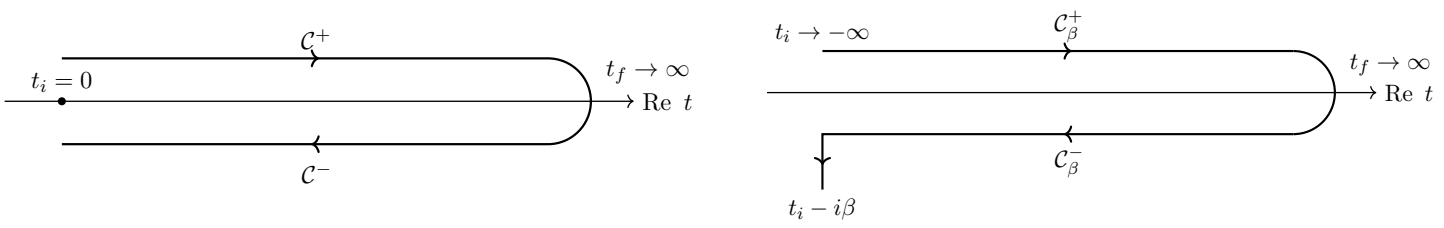

Figure 1. Non-equilibrium (left) and equilibrium (right) contours $\mathcal{C}$ in the complex time plane.

size as the initial one $[25] .^{1}$ Since one can neglect the Hubble expansion, i.e. assume a constant temperature, during the first stage, we will for technical convenience follow [18] in calculating this initial lepton asymmetry at fixed $T$ starting from zero initial Majorana neutrino abundance. In reality, any lepton asymmetry gets continually converted to a baryon asymmetry by sphaleron processes, which are in equilibrium for temperatures above the electroweak phase transition. Our first calculation of gauge corrections focuses on the lepton aymmetry only and neglects these and other spectator processes, which may affect the final baryon asymmetry by $\sim 50 \%[26-28]$.

\subsection{Correlation functions and Kadanoff-Baym equations}

In a quantum field theoretical description of leptogenesis, the desired information is encoded in the non-equilibrium correlation function of the Majorana neutrino, which is weakly coupled to a thermal bath of SM particles. This requires the real time formalism of thermal quantum field theory [29], in which two-point Green functions live on a contour $\mathcal{C}$ in the complex time plane, whose form depends on whether the field is in equilibrium or not, cf. figure 1. Our formulation and notation follows [18], where details of the calculation can be found. Green functions can be expressed in terms of Wightman functions, which for a real scalar field read

$$
\begin{aligned}
\Delta_{\mathcal{C}}\left(x_{1}, x_{2}\right) & =\theta_{\mathcal{C}}\left(x_{1}^{0}, x_{2}^{0}\right) \Delta^{>}\left(x_{1}, x_{2}\right)+\theta_{\mathcal{C}}\left(x_{2}^{0}, x_{1}^{0}\right) \Delta^{<}\left(x_{1}, x_{2}\right) \\
\Delta^{>}\left(x_{1}, x_{2}\right) & =\left\langle\phi\left(x_{1}\right) \phi\left(x_{2}\right)\right\rangle \\
\Delta^{<}\left(x_{1}, x_{2}\right) & =\left\langle\phi\left(x_{2}\right) \phi\left(x_{1}\right)\right\rangle
\end{aligned}
$$

with the Heaviside $\theta_{\mathcal{C}}$-function enforcing path ordering along the time contour $\mathcal{C}$. These in turn are related to the spectral function and statistical propagator, respectively,

$$
\begin{aligned}
& \Delta^{-}\left(x_{1}, x_{2}\right)=\mathrm{i}\left\langle\left[\phi\left(x_{1}\right), \phi\left(x_{2}\right)\right]\right\rangle=\mathrm{i}\left(\Delta^{>}\left(x_{1}, x_{2}\right)-\Delta^{<}\left(x_{1}, x_{2}\right)\right), \\
& \Delta^{+}\left(x_{1}, x_{2}\right)=\frac{1}{2}\left\langle\left\{\phi\left(x_{1}\right), \phi\left(x_{2}\right)\right\}\right\rangle=\frac{1}{2}\left(\Delta^{>}\left(x_{1}, x_{2}\right)+\Delta^{<}\left(x_{1}, x_{2}\right)\right) .
\end{aligned}
$$

Analogous quantities are defined for the Green functions of leptons and the heavy Majorana neutrino, with the role of (anti-)commutators exchanged for fermions,

$$
\begin{aligned}
S_{\alpha \beta}^{-}\left(x_{1}, x_{2}\right) & =\mathrm{i}\left\langle\left\{l_{L, \alpha}\left(x_{1}\right), \bar{l}_{L, \beta}\left(x_{2}\right)\right\}\right\rangle, & S_{\alpha \beta}^{+}\left(x_{1}, x_{2}\right) & =\frac{1}{2}\left\langle\left[l_{L, \alpha}\left(x_{1}\right), \bar{l}_{L, \beta}\left(x_{2}\right)\right]\right\rangle, \\
G_{\alpha \beta}^{-}\left(x_{1}, x_{2}\right) & =\mathrm{i}\left\langle\left\{N_{\alpha}\left(x_{1}\right), \bar{N}_{\beta}\left(x_{2}\right)\right\}\right\rangle, & G_{\alpha \beta}^{+}\left(x_{1}, x_{2}\right) & =\frac{1}{2}\left\langle\left[N_{\alpha}\left(x_{1}\right), \bar{N}_{\beta}\left(x_{2}\right)\right]\right\rangle .
\end{aligned}
$$

\footnotetext{
${ }^{1} \mathrm{~A}$ final lepton asymmetry of similar size is also generated when starting from a non-zero Majorana neutrino abundance [25].
} 
In the following, $\Pi_{\mathcal{C}}$ denotes the self-energy of the leptons and $\Sigma_{\mathcal{C}}$ that of the Majorana neutrino. These are equally defined on the time contour and can be similarly decomposed into Wightman functions,

$$
\Sigma_{\mathcal{C}}\left(x_{1}, x_{2}\right)=\theta_{\mathcal{C}}\left(x_{1}^{0}, x_{2}^{0}\right) \Sigma^{>}\left(x_{1}, x_{2}\right)+\theta_{\mathcal{C}}\left(x_{2}^{0}, x_{1}^{0}\right) \Sigma^{<}\left(x_{1}, x_{2}\right) .
$$

According to the physics scenario given in section 2.1, the non-equilibrium Green function of the Majorana neutrino has to be evaluated on the Schwinger-Keldysh contour, figure 1 (left), while the lepton and Higgs fields are in the thermal bath of SM particles described by the equilibrium contour, figure 1 (right), with inverse temperature $\beta=1 / T$. The full time evolution of the Majorana neutrino correlation function is given by the coupled set of Kadanoff-Baym equations,

$$
\begin{aligned}
& C\left(\mathrm{i} \gamma^{0} \partial_{t_{1}}-\mathbf{p} \boldsymbol{\gamma}-M\right) G_{\mathbf{p}}^{-}\left(t_{1}, t_{2}\right) \\
& \quad+\int_{t_{1}}^{t_{2}} \mathrm{~d} t^{\prime} C \Sigma_{\mathbf{p}}^{-}\left(t_{1}, t^{\prime}\right) G_{\mathbf{p}}^{-}\left(t^{\prime}, t_{2}\right)=0, \\
& C\left(\mathrm{i} \gamma^{0} \partial_{t_{1}}-\mathbf{p} \gamma-M\right) G_{\mathbf{p}}^{+}\left(t_{1}, t_{2}\right) \\
& \quad-\int_{t_{i}}^{t_{1}} \mathrm{~d} t^{\prime} C \Sigma_{\mathbf{p}}^{-}\left(t_{1}, t^{\prime}\right) G_{\mathbf{p}}^{+}\left(t^{\prime}, t_{2}\right)=-\int_{t_{i}}^{t_{2}} \mathrm{~d} t^{\prime} C \Sigma_{\mathbf{p}}^{+}\left(t_{1}, t^{\prime}\right) G_{\mathbf{p}}^{-}\left(t^{\prime}, t_{2}\right),
\end{aligned}
$$

with the spatial Fourier transforms assuming spatial homogeneity $\left(\mathbf{x}=\mathbf{x}_{1}-\mathbf{x}_{2}\right)$,

$$
\begin{aligned}
& G_{\mathbf{p}}^{ \pm}\left(t_{1}, t_{2}\right)=\int \mathrm{d}^{3} x \mathrm{e}^{-\mathrm{i} \mathbf{p x}} G^{ \pm}\left(t_{1}, t_{2}, \mathbf{x}\right), \\
& \Sigma_{\mathbf{p}}^{ \pm}\left(t_{1}, t_{2}\right)=\int \mathrm{d}^{3} x \mathrm{e}^{-\mathrm{i} \mathbf{p x}} \Sigma^{ \pm}\left(t_{1}, t_{2}, \mathbf{x}\right) .
\end{aligned}
$$

These equations are exact and in particular contain all quantum effects. Interactions with the plasma are automatically included via the self-energies $\Sigma^{ \pm}\left(x_{1}, x_{2}\right)$, as can be seen via generalised cutting rules. The leading-order contribution to the self-energy is given by the diagram in figure 2 .

An analytic solution can be obtained if the Yukawa coupling of the Majorana neutrino to the thermal bath is weak, so that back-reactions, being of higher order and additionally suppressed by the large number of degrees in freedom of the bath, can be neglected, and a narrow-width approximation $\Gamma \sim \lambda^{2} T \ll T$ is justified [18]. ${ }^{2}$ Since the particles in the loop are thermal, the heavy neutrino spectral function is time-translation invariant,

$$
G_{\mathbf{p}}^{-}\left(t_{1}, t_{2}\right)=G_{\mathbf{p}}^{-}\left(t_{1}-t_{2}\right)
$$

Using a zero abundance of the Majorana neutrinos at initial time $t_{i}=0$ and denoting

\footnotetext{
${ }^{2}$ Note that for $T<M$ one has $\Gamma \sim \lambda^{2} M \ll M$.
} 


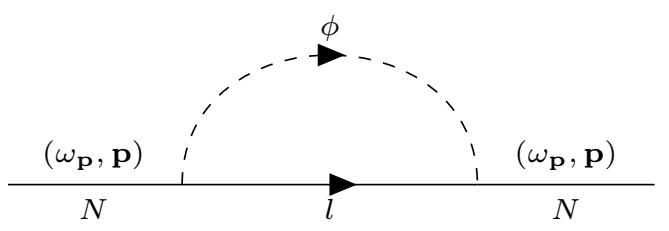

Figure 2. One-loop self-energy contribution to the Majorana neutrino self-energy $C \Sigma_{\mathbf{p}}^{ \pm}$.

$t_{12} \equiv\left(t_{1}+t_{2}\right), \Delta t_{12} \equiv\left(t_{1}-t_{2}\right)$, the solution for the Kadanoff-Baym equation is

$$
\begin{aligned}
G_{\mathbf{p}}^{-}\left(\Delta t_{12}\right)= & \left(\mathrm{i} \gamma_{0} \cos \left(\omega_{\mathbf{p}} \Delta t_{12}\right)+\frac{M-\mathbf{p} \boldsymbol{\gamma}}{\omega_{\mathbf{p}}} \sin \left(\omega_{\mathbf{p}} \Delta t_{12}\right)\right) \mathrm{e}^{-\Gamma_{\mathbf{p}}\left(\omega_{\mathbf{p}}\right)\left|\Delta t_{12}\right| / 2} C^{-1}, \\
G_{\mathbf{p}}^{+}\left(t_{12}, \Delta t_{12}\right)= & -\left(\mathrm{i} \gamma_{0} \sin \left(\omega_{\mathbf{p}} \Delta t_{12}\right)-\frac{M-\mathbf{p} \boldsymbol{\gamma}}{\omega_{\mathbf{p}}} \cos \left(\omega_{\mathbf{p}} \Delta t_{12}\right)\right) \\
& \times\left[\frac{1}{2} \tanh \left(\frac{\beta \omega_{\mathbf{p}}}{2}\right) \mathrm{e}^{-\Gamma_{\mathbf{p}}\left(\omega_{\mathbf{p}}\right)\left|\Delta t_{12}\right| / 2}+f_{F}\left(\omega_{\mathbf{p}}\right) \mathrm{e}^{-\Gamma_{\mathbf{p}}\left(\omega_{\mathbf{p}}\right) t_{12} / 2}\right] C^{-1},
\end{aligned}
$$

with $\omega_{\mathbf{p}}=\sqrt{\mathbf{p}^{2}+M^{2}}$ and the Fermi-Dirac distribution function $f_{F}\left(\omega_{\mathbf{p}}\right)$.

In general, $\Gamma_{\mathbf{p}}$ is the Majorana neutrino decay width resulting from its self-energy via

$$
\omega \Gamma_{\mathbf{p}}(\omega)=\frac{1}{4} \operatorname{tr}\left(-\mathrm{i} \not p \Sigma_{\mathbf{p}}^{-}(\omega)\right)
$$

At the level of the calculation in [18], this decay width does not include gauge corrections, and for its later use in the lepton number matrix is modeled by a momentum-independent constant. In section 3 we extend the calculation to include gauge corrections, and keep the full momentum-dependence in the lepton number matrix. Due to its appearance in the Majorana neutrino propagators, we will often need the on-shell value $\Gamma_{\mathbf{p}}\left(\omega_{\mathbf{p}}\right)$, which satisfies

$$
\Gamma_{\mathbf{p}}\left(\omega_{\mathbf{p}}\right)=\Gamma_{\mathbf{p}}\left(-\omega_{\mathbf{p}}\right)=\Gamma_{-\mathbf{p}}\left(\omega_{\mathbf{p}}\right)
$$

\subsection{The lepton number matrix}

A net lepton number density is obtained from the flavour-diagonal lepton number current

$$
\begin{aligned}
& n_{L}=\sum_{i} n_{L, i i}=\frac{1}{V} \int \mathrm{d}^{3} x \sum_{i} j_{i i}^{0}(x), \\
& j_{i j}^{\mu}(x)=\bar{l}_{L i} \gamma^{\mu} l_{L j}=-\lim _{x^{\prime} \rightarrow x} \operatorname{tr}\left[\gamma^{\mu} S_{L, i j}^{+}\left(x, x^{\prime}\right)\right] .
\end{aligned}
$$

The lepton number matrix is defined as the (spatial) Fourier transform of the current's zero component,

$$
L_{\mathbf{k}, i j}(t)=-\operatorname{tr}\left[\gamma^{0} S_{L, \mathbf{k}, i j}^{+}\left(t, t^{\prime}\right)\right]_{t \rightarrow t^{\prime}} .
$$

It has to be evaluated calculating the leading order correction to the lepton statistical propagator, contributing the necessary $C P$-violation. The corresponding Feynman diagrams are shown in figure 3 , giving [18]

$$
L_{\mathbf{k}, i i}=12 \operatorname{Im}\left(\lambda_{i 1}^{*}\left(\eta \lambda^{*}\right)_{i 1}\right) \int_{0}^{t} \mathrm{~d} t_{1} \int_{0}^{t} \mathrm{~d} t_{2} \operatorname{Re}\left[\operatorname{tr}\left(\Pi_{\mathbf{k}}^{(1),>}\left(t_{1}, t_{2}\right) S_{\mathbf{k}}^{<}\left(t_{2}-t_{1}\right)\right)\right]
$$


with the lepton self-energy from the first graph ${ }^{3}$ in figure 3 ,

$$
\begin{aligned}
& \Pi_{\mathbf{k}}^{(1),>}\left(t_{1}, t_{2}\right)=\int_{0}^{\infty} \mathrm{d} t_{3} \int_{\mathbf{p}, \mathbf{q}, \mathbf{k}^{\prime}, \mathbf{q}^{\prime}}(2 \pi)^{3} \delta^{3}\left(\mathbf{p}-\mathbf{k}^{\prime}-\mathbf{q}^{\prime}\right)(2 \pi)^{3} \delta^{3}(\mathbf{p}+\mathbf{k}+\mathbf{q}) \\
& \quad \times\left[\tilde{G}_{\mathbf{p}}\left(t_{1}, t_{3}\right) \theta\left(\Delta t_{23}\right)\left(S_{\mathbf{k}^{\prime}}^{>}\left(\Delta t_{23}\right) \Delta_{\mathbf{q}^{\prime}}^{>}\left(\Delta t_{23}\right)-S_{\mathbf{k}^{\prime}}^{<}\left(\Delta t_{23}\right) \Delta_{\mathbf{q}^{\prime}}^{<}\left(\Delta t_{23}\right)\right) \Delta_{\mathbf{q}}^{<}\left(\Delta t_{21}\right) P_{L}\right] .
\end{aligned}
$$

Here we introduced the notation $\int_{\mathbf{p}} \equiv \int \mathrm{d}^{3} p /(2 \pi)^{3}$ and $\tilde{G}_{\mathbf{p}}$ is the scalar non-equilibrium part of the Majorana neutrino propagator, connected to the full propagator via projections

$$
P_{L} G_{\mathbf{p}}\left(t_{1}, t_{2}\right) C P_{L}=P_{L} G_{\mathbf{p}}^{\mathrm{eq}}\left(t_{1}-t_{2}\right) C P_{L}+\tilde{G}_{\mathbf{p}}\left(t_{1}, t_{2}\right) P_{L}
$$

with the solution

$$
\tilde{G}_{\mathbf{p}}\left(t_{1}, t_{2}\right)=\frac{M}{\omega_{\mathbf{p}}} \cos \left(\omega_{\mathbf{p}} \Delta t_{12}\right) f_{F}\left(\omega_{\mathbf{p}}\right) \mathrm{e}^{-\Gamma_{\mathbf{p}} t_{12} / 2} .
$$

Note that the equilibrium part of the neutrino propagator does not contribute. We do not need and hence do not give the explicit form of the Higgs and lepton propagators $\Delta_{\mathbf{q}}^{\lessgtr}$ and $S_{\mathrm{k}}^{\lessgtr}$, which can however be found in [18]. Using the relations between the different kinds of propagators [29] and identifying

$$
\Sigma_{\mathbf{k}^{\prime}, \mathbf{q}^{\prime}}^{\lessgtr}\left(\Delta t_{23}\right)=S_{\mathbf{k}^{\prime}}^{\lessgtr}\left(\Delta t_{23}\right) \Delta_{\mathbf{q}^{\prime}}^{\lessgtr}\left(\Delta t_{23}\right), \quad \Sigma_{\mathbf{k}, \mathbf{q}}^{<}(\omega)=-2 f_{F}(\omega) \operatorname{Im}\left(\Sigma_{\mathbf{k}, \mathbf{q}}^{\mathrm{ret}}(\omega)\right),
$$

it is possible to express the lepton number matrix in terms of the retarded Majorana neutrino self-energy $\Sigma^{\mathrm{ret}}$. Using $\operatorname{Im}\left(\lambda_{i 1}^{*}\left(\eta \lambda^{*}\right)_{i 1}\right)=16 \pi \epsilon_{i i} /(3 M)$, with $\epsilon_{i i}$ parametrising the strength of CP violation [30], we have

$$
\begin{aligned}
& L_{\mathbf{k}, i i}(t) \\
& =-\frac{256 \pi \epsilon_{i i}}{M} \int_{0}^{t} \mathrm{~d} t_{1} \int_{0}^{t} \mathrm{~d} t_{2} \int_{0}^{t_{2}} \mathrm{~d} t_{3} \int_{-\infty}^{\infty} \frac{\mathrm{d} \omega_{21}}{2 \pi} \int_{-\infty}^{\infty} \frac{\mathrm{d} \omega_{23}}{2 \pi} \int_{\mathbf{p}, \mathbf{k}^{\prime}, \mathbf{q}, \mathbf{q}^{\prime}}(2 \pi)^{6} \delta^{3}\left(\mathbf{p}-\mathbf{k}^{\prime}-\mathbf{q}^{\prime}\right) \delta^{3}(\mathbf{p}+\mathbf{k}+\mathbf{q}) \\
& \times \tilde{G}_{\mathbf{p}}\left(t_{1}, t_{3}\right) f_{F}\left(\omega_{21}\right) \operatorname{tr}\left(\operatorname{Im} \Sigma_{R, \mathbf{k}, \mathbf{q}}^{\mathrm{ret}}\left(\omega_{21}\right) \operatorname{Im} \Sigma_{L, \mathbf{k}^{\prime}, \mathbf{q}^{\prime}}^{\mathrm{ret}}\left(\omega_{23}\right)\right) \operatorname{Re}\left[\mathrm{e}^{\left.-\mathrm{i}\left(\omega_{21} \Delta t_{21}+\omega_{23} \Delta t_{23}\right)\right] .}\right.
\end{aligned}
$$

This expression contains quantum mechanical off-shell and memory effects. However, it does not yet contain corrections from interactions with SM gauge bosons, which introduce thermal damping and the associated widths for the Higgs and lepton fields. As explained in a detailed discussion in [18], this fact is responsible for the apparent difference between quantum Boltzmann solutions and the present solution of Kadanoff-Baym equations. Moreover, for $T \gtrsim M$ these damping widths are of order $\gamma_{l, \phi} \sim g_{\mathrm{SM}}^{2} T \gg \lambda^{2} T \sim \Gamma$, i.e. larger than the heavy neutrino rate, and hence can modify the result significantly [19]. Any quantitative theory of quantum leptogenesis therefore requires a systematic inclusion of these effects.

\section{Gauge corrections to leptogenesis}

The inclusion of gauge corrections to thermal leptogenesis is complicated by the fact that it happens before the electroweak transition, i.e. gauge bosons are massless and the nonabelian weak dynamics are QCD-like [31-33]. This in particular implies the dynamical

\footnotetext{
${ }^{3}$ The contribution of the second graph can be expressed in terms of the first one.
} 

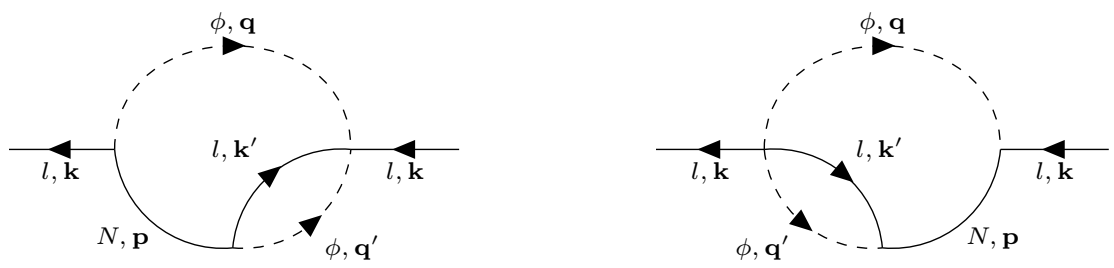

Figure 3. Two-loop corrections to the lepton self-energy $\Pi^{ \pm}$leading to non-zero lepton number densities.

generation of mass scales $\sim T, g T, g^{2} T$, of which the ultra-soft $\sim g^{2} T$ is entirely nonperturbative. For processes with gauge particles on the soft scale $\sim g T$, hard thermal loops (HTL) contribute the same power in the coupling constant to the self-energy to any loop order, and hence have to be resummed when counting orders in the gauge couplings [29, 34]. Here we consider fermion processes, whose external momenta in a plasma are typically hard, $k \sim T$. Nevertheless, for hard momenta near the light cone, $k^{2} \sim g_{\mathrm{SM}}^{2} T^{2}$, loop momenta collinear with the external ones require a similar resummation of collinear thermal loops (CTL) [35]. For power counting purposes, all SM couplings are considered as parametrically the same.

\subsection{CTL resummation of the Majorana neutrino self-energy}

As a first step, we need the gauge corrections to the Majorana neutrino self-energy and its proper resummation shown in figure 4 to determine the Majorana neutrino production rate [20]. One effect of interactions with the plasma is to modify the dispersion relations of the thermalised leptons and Higgs particles by the asymptotic thermal masses ${ }^{4}$

$$
\begin{aligned}
m_{\phi, \infty} & =\frac{1}{16}\left(3 g^{2}+g^{\prime 2}+4 h_{t}^{2}+8 \lambda_{\phi}\right) T^{2}, \\
m_{l, \infty} & =\frac{1}{16}\left(3 g^{2}+g^{\prime 2}\right) T^{2} .
\end{aligned}
$$

Next, we consider nearly collinear momenta $k$ close to the light cone, pointing approximately in the three-direction of the light-like four-vector $v=(1, \mathbf{v}), \mathbf{v}^{2}=1$. Three-momenta are then specified by components parallel and perpendicular to $\mathbf{v}$,

$$
\mathbf{k}=k_{\|} \hat{\mathbf{v}}+\mathbf{k}_{\perp}, \quad \text { with } \quad k_{\|}:=\mathbf{k} \cdot \mathbf{v}, \quad \mathbf{k}_{\perp} \cdot \mathbf{v}=0
$$

The leptonic and Higgs loop momenta thus satisfy

$$
k_{\|} \sim T, \quad\left|\mathbf{k}_{\perp}\right| \sim g_{\mathrm{SM}} T, \quad v \cdot k \sim g_{\mathrm{SM}}^{2} T, \quad k^{2} \sim g_{\mathrm{SM}}^{2} T^{2},
$$

and thermal masses are important. Further, the gauge loop momenta are considered soft, $u_{\mu} \sim g_{\mathrm{SM}} T$.

\footnotetext{
${ }^{4}$ Since other Yukawa couplings are very small, we include only the coupling of the top quark.
} 

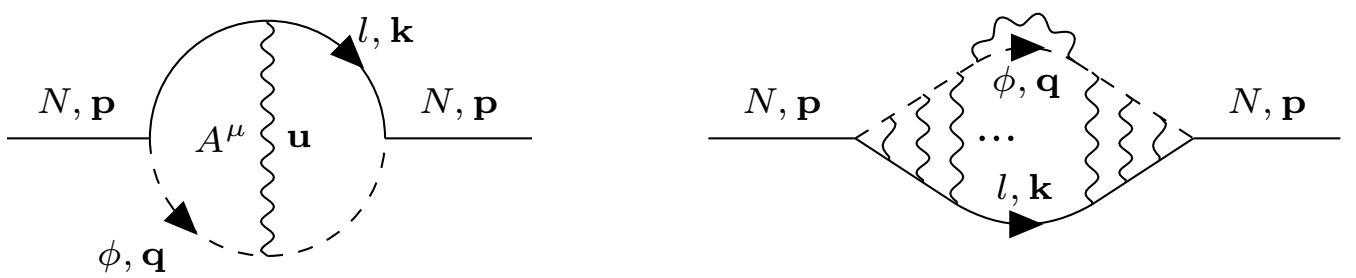

Figure 4. Majorana neutrino self-energy contributions with one and several additional gauge bosons with soft three-momentum $\mathbf{u} \sim g_{\mathrm{SM}} T$.

In this kinematical region the diagrams without and with gauge corrections, figures 2 and 4, respectively, are parametrically the same,

$$
\begin{aligned}
& \Sigma_{1 \text {-loop }} \sim \underbrace{g_{\text {SM }}^{2}}_{\text {3-vertex factor }} \underbrace{\left(\frac{1}{g_{\mathrm{SM}}^{2}}\right)^{2}}_{\text {propagators }} \underbrace{g_{\text {SM }}^{4}}_{\text {phase }} \sim g_{\text {Space integration }}^{2}, \\
& \Sigma_{\text {2-loop }} \sim \underbrace{g_{\mathrm{SM}}^{2}}_{\text {3-vertex factor }} \underbrace{g_{\mathrm{SM}}^{2}}_{\text {gauge boson vertices }} \underbrace{\left(\frac{1}{g_{\mathrm{SM}}^{2}}\right)^{5}}_{\text {propagators }} \underbrace{\left(g_{\mathrm{SM}}^{4}\right)^{2}}_{\text {phase space integration }} \sim g_{\mathrm{SM}}^{2} .
\end{aligned}
$$

The 3-vertex factor in each expression arises kinematically, where we followed the power counting rules from $[20,35]$. Thus, adding soft internal gauge boson lines does not increase the order in the gauge coupling and necessitates a resummation of the entire ladder to infinite loop order, figure 4, while contributions from crossed ladder rungs and vertex corrections to loop particles are further suppressed [20, 35].

The problem corresponds to the Landau-Pomeranchuk-Migdal effect and has also been solved for gluon radiation in a QCD plasma [36]. Its application to the heavy Majorana neutrino was treated in [20], where the ladder-resummed self-energy, including thermals widths of the Higgs and leptons, is written as

$$
\Sigma_{R, \mathbf{p}}^{\mathrm{ret}}(\omega)=-\lambda^{2} \frac{\mathrm{i}}{2} \int_{\mathbf{k}} \frac{d(r) \mathcal{F}\left(p_{\|}, k_{\|}\right)}{k_{\|}-p_{\|}}\left(\begin{array}{c}
-\frac{k_{1}-\mathrm{i} k_{2}}{2 k_{\|}} \\
1
\end{array}\right) \cdot\left(-\frac{f^{1}+\mathrm{i} f^{2}}{4 k_{\|}}, \psi\right)
$$

with $d(r)=2$ the dimension of the $\mathrm{SU}(2)$ fundamental representation and the combination of Fermi-Dirac and Bose-Einstein distributions

$$
\mathcal{F}\left(p_{\|}, k_{\|}\right)=f_{F}\left(k_{\|}\right)+f_{B}\left(k_{\|}-p_{\|}\right) .
$$

The functions $\psi$ and $\mathbf{f}=\left(f^{1}, f^{2}\right)$ are defined as solutions of the integral equations

$$
\begin{array}{r}
\mathrm{i} \epsilon(p, \mathbf{k}) \mathbf{f}\left(\mathbf{k}_{\perp}\right)-\int \frac{\mathrm{d}^{2} u_{\perp}}{(2 \pi)^{2}} \mathcal{C}\left(\left|\mathbf{u}_{\perp}\right|\right)\left[\mathbf{f}\left(\mathbf{k}_{\perp}\right)-\mathbf{f}\left(\mathbf{k}_{\perp}-\mathbf{u}_{\perp}\right)\right]=2 \mathbf{k}_{\perp}, \\
\mathrm{i} \epsilon(p, \mathbf{k}) \psi\left(\mathbf{k}_{\perp}\right)-\int \frac{\mathrm{d}^{2} u_{\perp}}{(2 \pi)^{2}} \mathcal{C}\left(\left|\mathbf{u}_{\perp}\right|\right)\left[\psi\left(\mathbf{k}_{\perp}\right)-\psi\left(\mathbf{k}_{\perp}-\mathbf{u}_{\perp}\right)\right]=1,
\end{array}
$$

with the kernel

$$
\mathcal{C}\left(\left|\mathbf{u}_{\perp}\right|\right)=T\left[C_{2}(r) g^{2}\left(\frac{1}{\left|\mathbf{u}_{\perp}\right|^{2}}-\frac{1}{\left|\mathbf{u}_{\perp}\right|^{2}+m_{D}^{2}}\right)+y_{l}^{2} g^{\prime 2}\left(\frac{1}{\left|\mathbf{u}_{\perp}\right|^{2}}-\frac{1}{\left|\mathbf{u}_{\perp}\right|^{2}+m_{D}^{\prime 2}}\right)\right] .
$$


The kernel is obtained from the gauge field propagator and contains the ladder resummation, with the $\mathrm{SU}(2)$ Casimir operator $C_{2}(r)=3 / 4$, the hypercharge $y_{l}=-1 / 2$, and the HTL-resummed Debye masses [37]

$$
m_{D}^{2}=\frac{11}{6} g^{2} T^{2}, \quad m_{D}^{\prime 2}=\frac{11}{6} g^{\prime 2} T^{2} .
$$

The quantity $\epsilon(p, \mathbf{k})$ is given in a frame, where $\mathbf{v} \| \mathbf{p}$ and $\mathbf{p}_{\perp}=0$, as

$$
\epsilon\left(\omega, p_{\|}, \mathbf{k}\right)=\alpha\left(\omega, p_{\|}, \mathbf{k}\right)+\beta\left(p_{\|}, k_{\|}\right) \mathbf{k}_{\perp}^{2}=\beta\left(p_{\|}, k_{\|}\right)\left(M_{\mathrm{eff}}^{2}+\mathbf{k}_{\perp}^{2}\right)
$$

with

$$
\begin{aligned}
\alpha\left(\omega, p_{\|}, k_{\|}\right) & =\omega-p_{\|}+\frac{m_{\phi, \infty}^{2}}{2\left(k_{\|}-p_{\|}\right)}-\frac{m_{l, \infty}^{2}}{2 k_{\|}}, \\
\beta\left(p_{\|}, k_{\|}\right) & =\frac{p_{\|}}{2 k_{\|}\left(k_{\|}-p_{\|}\right)}, \\
M_{\text {eff }}^{2} & =\frac{m_{l, \infty}^{2}\left(p_{\|}-k_{\|}\right)+m_{\phi, \infty}^{2} k_{\|}+2 k_{\|}\left(\omega-p_{\|}\right)\left(k_{\|}-p_{\|}\right)}{p_{\|}} .
\end{aligned}
$$

The integral eqs. (3.7), (3.8) are best solved in Fourier space,

$$
\psi(\mathbf{b})=\int \frac{\mathrm{d}^{2} k_{\perp}}{(2 \pi)^{2}} \psi\left(\mathbf{k}_{\perp}\right) \mathrm{e}^{\mathrm{i} \mathbf{k}_{\perp} \mathbf{b}}, \quad \mathbf{f}(\mathbf{b})=\int \frac{\mathrm{d}^{2} k_{\perp}}{(2 \pi)^{2}} \mathbf{f}\left(\mathbf{k}_{\perp}\right) \mathrm{e}^{\mathrm{i} \mathbf{k}_{\perp} \mathbf{b}} .
$$

Rotational invariance implies $\psi(\mathbf{b})=\psi(b), \mathbf{f}(\mathbf{b})=h(b) \mathbf{b}$, and the equations are converted into ordinary differential equations,

$$
\begin{aligned}
& -\mathrm{i} \beta\left(\partial_{b}^{2}+\frac{1}{b} \partial_{b}-M_{\text {eff }}^{2}\right) \psi(b)-\mathcal{K}(b) \psi(b)=0, \\
& -\mathrm{i} \beta\left(\partial_{b}^{2}+\frac{3}{b} \partial_{b}-M_{\text {eff }}^{2}\right) h(b)-\mathcal{K}(b) h(b)=0,
\end{aligned}
$$

with $\mathcal{K}(b)$ the Fourier transform of $\mathcal{C}\left(\mathbf{u}_{\perp}\right)^{5}$

$$
\mathcal{K}(b)=\frac{1}{(2 \pi)^{2}} \int_{0}^{2 \pi} \mathrm{d} \phi \int_{0}^{\infty} \mathrm{d}\left|\mathbf{u}_{\perp}\right|\left(1-\mathrm{e}^{\mathrm{i}\left|\mathbf{u}_{\perp}\right| b \cos (\phi)}\right)\left|\mathbf{u}_{\perp}\right| \mathcal{C}\left(\left|\mathbf{u}_{\perp}\right|\right) .
$$

The solution of eqs. (3.16), (3.17) determines the Majorana neutrino self-energy in the form

$$
\begin{aligned}
\operatorname{Im}\left(\Sigma_{R, \mathbf{p}}^{\mathrm{ret}}(\omega)\right) & =-\frac{\lambda^{2} d(r)}{2} \int \frac{\mathrm{d} k_{\|}}{2 \pi} \frac{\mathcal{F}\left(p_{\|}, k_{\|}\right)}{k_{\|}-p_{\|}}\left(\begin{array}{cc}
\frac{\operatorname{Im}\left(c_{2, h}\left(\omega, p_{\|}, k_{\|}\right)\right)}{4 k_{\|}^{2}} & 0 \\
0 & \operatorname{Re}\left(c_{2, \psi}\left(\omega, p_{\|}, k_{\|}\right)\right)
\end{array}\right) \\
& \equiv-\frac{\lambda^{2} d(r)}{2}\left(\begin{array}{cc}
\sigma_{h}\left(\omega, p_{\|}\right) & 0 \\
0 & \sigma_{\psi}\left(\omega, p_{\|}\right)
\end{array}\right),
\end{aligned}
$$

\footnotetext{
${ }^{5}$ The calculation of $\mathcal{K}(b)$ requires a renormalization procedure to perform the Fourier transformation as well as the $\mathbf{u}_{\perp}$ integral. A detailed calculation can be found in [20].
} 


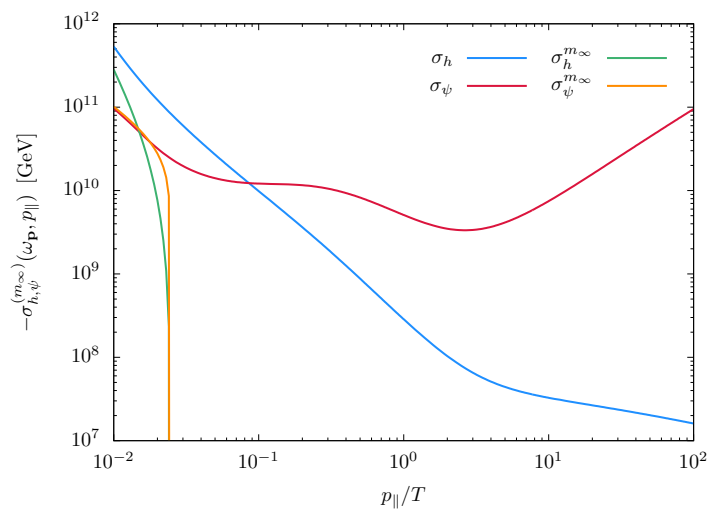

Figure 5. The fully gauge corrected $\sigma_{h, \psi}\left(\omega_{\mathbf{p}}, p_{\|}\right)$and the $\sigma_{h, \psi}^{m_{\infty}}\left(\omega_{\mathbf{p}}, p_{\|}\right)$, including asymptotic masses only, evaluated on-shell, $\omega=\omega_{\mathbf{p}}$, with $M=10^{10} \mathrm{GeV}$ and $T=10^{11} \mathrm{GeV}$.

where the $c_{2}$ are asymptotic solutions

$$
\begin{gathered}
\int \frac{\mathrm{d}^{2} k_{\perp}}{(2 \pi)^{2}} \operatorname{Re}\left(\mathbf{k}_{\perp} \cdot \mathbf{f}_{\perp}\left(\mathbf{k}_{\perp}\right)\right)=\lim _{b \rightarrow 0} \operatorname{Im}(2 h(b)) \equiv 2 \operatorname{Im}\left(c_{2, h}\left(\omega, p_{\|}, k_{\|}\right)\right), \\
\int \frac{\mathrm{d}^{2} k_{\perp}}{(2 \pi)^{2}} \operatorname{Re}\left(\psi\left(\mathbf{k}_{\perp}\right)\right)=\lim _{b \rightarrow 0} \operatorname{Re}(\psi(b)) \equiv \operatorname{Re}\left(c_{2, \psi}\left(\omega, p_{\|}, k_{\|}\right)\right) .
\end{gathered}
$$

The off-diagonal elements of eq. (3.19) receive contributions vanishing in the limit $b \rightarrow 0$, and $\mathcal{O}(1 / b)$ divergent contributions. These are removed by renormalization, since they appear in the temperature independent part of the self-energy, while the temperature dependent part is given by the function $\mathcal{F}\left(p_{\|}, k_{\|}\right)$. For later use in the lepton number density we define the functions

$$
\begin{aligned}
\sigma_{h}\left(\omega, p_{\|}\right) & \equiv \int \frac{\mathrm{d} k_{\|}}{2 \pi} \frac{\mathcal{F}\left(p_{\|}, k_{\|}\right)}{4 k_{\|}^{2}\left(k_{\|}-p_{\|}\right)} \operatorname{Im}\left(c_{2, h}\left(\omega, p_{\|}, k_{\|}\right)\right), \\
\sigma_{\psi}\left(\omega, p_{\|}\right) & \equiv \int \frac{\mathrm{d} k_{\|}}{2 \pi} \frac{\mathcal{F}\left(p_{\|}, k_{\|}\right)}{k_{\|}-p_{\|}} \operatorname{Re}\left(c_{2, \psi}\left(\omega, p_{\|}, k_{\|}\right)\right) .
\end{aligned}
$$

Because of the symmetry between right- and left-handed Dirac components, we have

$$
\begin{aligned}
\Sigma_{R, \mathbf{p}}^{\mathrm{ret}}(\omega) & =\Sigma_{L,-\mathbf{p}}^{\mathrm{ret}}(\omega), \\
\sigma_{h}\left(-\omega, p_{\|}\right) & =\sigma_{\psi}\left(\omega, p_{\|}\right), \quad \sigma_{h}\left(\omega,-p_{\|}\right)=\sigma_{\psi}\left(\omega, p_{\|}\right) .
\end{aligned}
$$

In order to quantify the effect of the ladder resummation, it is useful to also analyse eqs. (3.7), (3.8) with $\mathcal{C}\left(\left|\mathbf{u}_{\perp}\right|\right)=0$, giving a Majorana neutrino self-energy which is only partly corrected by asymptotic masses. The equations in this limit can be solved trivially and one obtains after $\mathbf{k}_{\perp}$-integration

$$
\begin{aligned}
\sigma_{h}^{m_{\infty}}\left(\omega, p_{\|}\right) & =\int_{k_{\text {low }}}^{k_{\text {high }}} \frac{d k_{\|}}{2 \pi} \frac{\mathcal{F}\left(p_{\|}, k_{\|}\right)}{k_{\|}-p_{\|}} \frac{\alpha\left(\omega, p_{\|}, k_{\|}\right)}{16 k_{\|}^{2} \beta\left(p_{\|}, k_{\|}\right)\left|\beta\left(p_{\|}, k_{\|}\right)\right|} \\
\sigma_{\psi}^{m_{\infty}}\left(\omega, p_{\|}\right) & =-\int_{k_{\text {low }}}^{k_{\text {high }}} \frac{d k_{\|}}{2 \pi} \frac{\mathcal{F}\left(p_{\|}, k_{\|}\right)}{k_{\|}-p_{\|}} \frac{1}{4\left|\beta\left(p_{\|}, k_{\|}\right)\right|}
\end{aligned}
$$



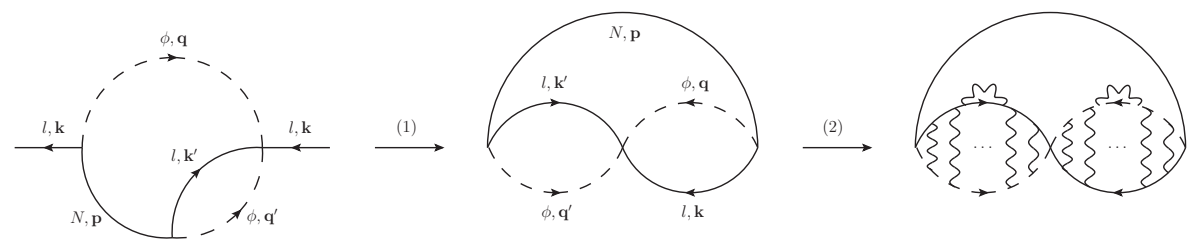

Figure 6. Transformation of the lepton self-energy diagram to a form amenable to resummation.

The integration boundaries in this case are given by

$$
k_{\text {low }}=\frac{X-\sqrt{Y}}{4\left(\omega-p_{\|}\right)}, \quad k_{\text {high }}=\frac{X+\sqrt{Y}}{4\left(\omega-p_{\|}\right)},
$$

with

$$
X \equiv m_{l, \infty}^{2}-m_{\phi, \infty}^{2}+2\left(\omega-p_{\|}\right) p_{\|}, \quad Y \equiv X^{2}-8 m_{l, \infty}^{2}\left(\omega-p_{\|}\right) p_{\|}, \quad Y \geq 0
$$

In figure 5 the fully corrected $\sigma_{h, \psi}\left(\omega_{\mathbf{p}}, p_{\|}\right)$are plotted for $M=10^{10} \mathrm{GeV}, T=$ $10^{11} \mathrm{GeV}$, and compared to the $\sigma_{h, \psi}^{m_{\infty}}\left(\omega_{\mathbf{p}}, p_{\|}\right)$. The latter are significantly smaller than their fully resummed counterparts in most of the parameter space considered. Note that above a certain $p_{\|}$, the $\sigma_{h, \psi}^{m_{\infty}}\left(\omega_{\mathbf{p}}, p_{\|}\right)$vanish kinematically.

For the SM corrected Majorana decay width we start with eq. (2.19). In Weyl basis it is possible to simplify further,

$$
\begin{aligned}
\Gamma_{\mathbf{p}}(\omega) & =\frac{1}{2 \omega} \operatorname{tr}\left[\not p \operatorname{Im}\left(\Sigma_{\mathbf{p}}^{\mathrm{ret}}(\omega)\right)\right] \\
& =\frac{1}{2 \omega} \operatorname{tr}\left[\left(\begin{array}{cc}
0 & \sigma \cdot p \\
\bar{\sigma} \cdot p & 0
\end{array}\right) \cdot\left(\begin{array}{cc}
0 & \operatorname{Im}\left(\Sigma_{R, \mathbf{p}}^{\mathrm{ret}}(\omega)\right) \\
\operatorname{Im}\left(\Sigma_{L, \mathbf{p}}^{\mathrm{ret}}(\omega)\right) & 0
\end{array}\right)\right] .
\end{aligned}
$$

Using $\mathbf{p}_{\perp}=0$ we find

$$
\Gamma_{\mathbf{p}}(\omega)=-\frac{\lambda^{2} d(r)}{2 \omega}\left(\left(\omega+p_{\|}\right) \sigma_{h}\left(\omega, p_{\|}\right)+\left(\omega-p_{\|}\right) \sigma_{\psi}\left(\omega, p_{\|}\right)\right)
$$

For all further calculations we only need the on-shell expression $\Gamma_{\mathbf{p}}=\Gamma_{\mathbf{p}}\left(\omega_{\mathbf{p}}\right)$ in the Majorana neutrino propagator, see eq. (2.27). Note that the symmetry property for the $\sigma_{h, \psi}$-functions (3.25) reflects the symmetry of $\Gamma_{\mathbf{p}}$, eq. (2.20).

\subsection{Gauge corrections to lepton self-energies}

We now have everything at hand to also include gauge corrections to the $C P$-violating diagrams in figure 3, which enter the lepton number matrix. Adding one internal gauge boson line modifies those diagrams by either self-energy or vertex corrections of the internal lines, the resulting three-loop diagrams are collected in appendix A. From the last section it is clear that in the collinear kinematical region this will not suffice for a result to leading order in the gauge coupling. Instead, infinitely many insertions have to be resummed. 
In order to achieve this, we follow the procedure depicted in figure 6 and first close the external fermion lines (1), which implies an integration over lepton momenta and transforms the lepton number matrix into the lepton number density,

$$
\int \frac{\mathrm{d}^{3} k}{(2 \pi)^{3}} L_{\mathbf{k}, i i}=n_{L, i i}
$$

In this form of the diagram, we can focus on the neutrino self-energy and apply the resummation from section 3.1. The only additional feature is the effective four-point vertex appearing in this self-energy, so that two of the resummed blobs from 3.1 are needed in step (2). It is instructive to check by direct comparison with appendix A that indeed all parametrically leading three-loop diagrams shown there are included and resummed. In appendix B we give a more detailed derivation of the double-blob diagram, which is general and also applies away from the hierarchical mass scenario.

\subsection{Lepton number matrix with complete SM corrections}

Collecting all results from the previous sections it is possible to give an expression for the SM corrected lepton number matrix. Starting with eq. (2.29) we first carry out the q and $\mathbf{q}^{\prime}$ momentum integrals by using the momentum conserving $\delta$-functions, so that the trace in the integrand takes the form

$$
\begin{aligned}
\int_{\mathbf{k}, \mathbf{k}^{\prime}} \operatorname{tr}\left(\operatorname{Im} \Sigma_{R, \mathbf{k},-\mathbf{p}-\mathbf{k}}^{\mathrm{ret}}\left(\omega_{21}\right) \operatorname{Im} \Sigma_{L, \mathbf{k}^{\prime}, \mathbf{p}-\mathbf{k}^{\prime}}^{\mathrm{ret}}\left(\omega_{23}\right)\right) & =\operatorname{tr}\left(\operatorname{Im} \Sigma_{R,-\mathbf{p}}^{\mathrm{ret}}\left(\omega_{21}\right) \operatorname{Im} \Sigma_{L, \mathbf{p}}^{\mathrm{ret}}\left(\omega_{23}\right)\right) \\
& =\operatorname{tr}\left(\operatorname{Im} \Sigma_{R, \mathbf{p}}^{\mathrm{ret}}\left(\omega_{21}\right) \operatorname{Im} \Sigma_{R, \mathbf{p}}^{\mathrm{ret}}\left(\omega_{23}\right)\right),
\end{aligned}
$$

where we have used the relation between the left- and right-handed self-energy (3.24).

Finally we use the resummed diagram figure 6 to insert the resummed Majorana neutrino self-energy in the equation above. Then, the trace can be carried out using eq. (3.19),

$$
\operatorname{tr}\left(\operatorname{Im} \Sigma_{R, \mathbf{p}}^{\mathrm{ret}}\left(\omega_{21}\right) \operatorname{Im} \Sigma_{R, \mathbf{p}}^{\mathrm{ret}}\left(\omega_{23}\right)\right)=\sigma_{\psi}\left(\omega_{21}, p_{\|}\right) \sigma_{\psi}\left(\omega_{23}, p_{\|}\right)+\sigma_{h}\left(\omega_{21}, p_{\|}\right) \sigma_{h}\left(\omega_{23}, p_{\|}\right) .
$$

Altogether, we arrive at the following result of the corrected lepton number density:

$$
\begin{aligned}
& n_{L, i i}(t)=-\frac{128}{\pi} \epsilon_{i i} \int_{0}^{t} \mathrm{~d} t_{1} \int_{0}^{t} \mathrm{~d} t_{2} \int_{0}^{t_{2}} \mathrm{~d} t_{3} \int_{0}^{\infty} \mathrm{d} p_{\|} \int_{-\infty}^{\infty} \frac{\mathrm{d} \omega_{21}}{2 \pi} \int_{-\infty}^{\infty} \frac{\mathrm{d} \omega_{23}}{2 \pi} \frac{p_{\|}^{2}}{\omega_{\mathbf{p}}} f_{F}\left(\omega_{\mathbf{p}}\right) f_{F}\left(\omega_{21}\right) \cos \left(\omega_{\mathbf{p}} \Delta t_{13}\right) \\
& \times \mathrm{e}^{-\Gamma_{\mathbf{p}} \frac{t_{13}}{2}} \operatorname{Re}\left(\mathrm{e}^{-\mathrm{i}\left(\omega_{21} \Delta t_{21}+\omega_{23} \Delta t_{23}\right)}\right)\left[\sigma_{\psi}\left(\omega_{21}, p_{\|}\right) \sigma_{\psi}\left(\omega_{23}, p_{\|}\right)+\sigma_{h}\left(\omega_{21}, p_{\|}\right) \sigma_{h}\left(\omega_{23}, p_{\|}\right)\right] .
\end{aligned}
$$

This expression contains all SM corrections to leading order in the respective couplings and for the first time gives a complete description of quantum leptogenesis.

\section{Evaluation of the lepton number density}

It now remains to numerically evaluate our final result eq. (3.36). As we shall see shortly, the time integrations can be performed analytically. However, the remaining three-dimensional integral has an integrand with rapidly oscillating parts, rendering an accurate numerical 
evaluation difficult. Moreover, $\sigma_{h}$ and $\sigma_{\psi}$ are specified by the asymptotic solutions of the differential eqs. (3.16) and (3.17), which have to be solved numerically for each call of those functions. A brute force numerical result would thus require a significant amount of computing power.

Here we adopt a different approach. Since the final result is accurate to leading order in the couplings only, we limit the integration range to those regions, which give the parametrically leading contribution. This permits us to do two more integrations analytically, leaving only the $p_{\|}$-integral as a final numerical task. Since we are left with only one momentum variable, we simplify the notation $p_{\|} \rightarrow p$ in this section.

\subsection{The time integrals}

We begin by factoring out the time integrals,

$$
\begin{aligned}
n_{L, i i}(t)= & -\frac{128}{\pi} \epsilon_{i i} \int_{0}^{\infty} \mathrm{d} p \int_{-\infty}^{\infty} \frac{\mathrm{d} \omega_{21}}{2 \pi} \int_{-\infty}^{\infty} \frac{\mathrm{d} \omega_{23}}{2 \pi} \mathcal{T}\left(t ; \omega_{21}, \omega_{23}, p\right) \frac{p^{2}}{\omega_{\mathbf{p}}} f_{F}\left(\omega_{\mathbf{p}}\right) f_{F}\left(\omega_{21}\right) \\
& \times\left[\sigma_{\psi}\left(\omega_{21}, p\right) \sigma_{\psi}\left(\omega_{23}, p\right)+\sigma_{h}\left(\omega_{21}, p\right) \sigma_{h}\left(\omega_{23}, p\right)\right]
\end{aligned}
$$

The resulting function,

$$
\mathcal{T}\left(t ; \omega_{21}, \omega_{23}, p\right) \equiv \int_{0}^{t} \mathrm{~d} t_{1} \int_{0}^{t} \mathrm{~d} t_{2} \int_{0}^{t_{2}} \mathrm{~d} t_{3} \cos \left(\omega_{\mathbf{p}} \Delta t_{13}\right) \mathrm{e}^{-\Gamma_{\mathbf{p}} \frac{t_{13}}{2}} \operatorname{Re}\left(\mathrm{e}^{-\mathrm{i}\left(\omega_{21} \Delta t_{21}+\omega_{23} \Delta t_{23}\right)}\right),
$$

can be integrated explicitly with the help of Mathematica, and the result is spelled out in appendix C. We now use this expression to estimate in which domain of frequencies it gives parametrically dominant contributions to the integral.

The denominator of (C.1) reads

$$
\begin{array}{r}
\left(\omega_{21}+\omega_{23}\right)\left(\Gamma_{\mathbf{p}}^{2}+4\left(\omega_{21}-\omega_{\mathbf{p}}\right)^{2}\right)\left(\Gamma_{\mathbf{p}}^{2}+4\left(\omega_{21}+\omega_{\mathbf{p}}\right)^{2}\right) \\
\times\left(\Gamma_{\mathbf{p}}^{2}+4\left(\omega_{23}-\omega_{\mathbf{p}}\right)^{2}\right)\left(\Gamma_{\mathbf{p}}^{2}+4\left(\omega_{23}+\omega_{\mathbf{p}}\right)^{2}\right) .
\end{array}
$$

At typical particle momentum in our system $p \sim \mathcal{O}(T)$, the Majorana neutrino decay width is of order $\Gamma_{\mathbf{p}} \lesssim \mathcal{O}\left(\lambda^{2} T\right)$, and since we are working at temperatures $T \gtrsim M$, this results in $\omega_{\mathbf{p}} \sim \mathcal{O}(T)$ as well. Hence in the four factors including $\Gamma_{\mathbf{p}}$, the latter is subdominant compared to the frequency terms. These factors are thus smallest when the two frequencies approach the mass shell. Alternatively, there is a pole due to the first factor. The function $\mathcal{T}$ thus has peaks in the following two limits, for which we estimate their parametric contribution:

1. $\omega_{21}, \omega_{23} \rightarrow \pm \omega_{\mathbf{p}}:$ symmetric under $\omega_{\mathbf{p}} \rightarrow-\omega_{\mathbf{p}}$,

$$
\begin{aligned}
\lim _{\omega_{23}, \omega_{21} \rightarrow \pm \omega_{\mathbf{p}}} \mathcal{T}\left(t ; \omega_{21}, \omega_{23}, p\right)= & \frac{2 \mathrm{e}^{-\Gamma_{\mathbf{p}} t}\left(\mathrm{e}^{\Gamma_{\mathbf{p}} t / 2}-1\right)}{\Gamma_{\mathbf{p}} \omega_{\mathbf{p}}\left(\Gamma_{\mathbf{p}}^{2}+16 \omega_{\mathbf{p}}^{2}\right)} \\
& \times\left[4 \omega_{\mathbf{p}}+\mathrm{e}^{\Gamma_{\mathbf{p}} t / 2}\left(-4 \omega_{\mathbf{p}} \cos \left(\omega_{\mathbf{p}} t\right)+\Gamma_{\mathbf{p}} \sin \left(2 \omega_{\mathbf{p}} t\right)\right)\right] \\
& \sim \mathcal{O}\left(\lambda^{-2} T^{-3}\right) .
\end{aligned}
$$




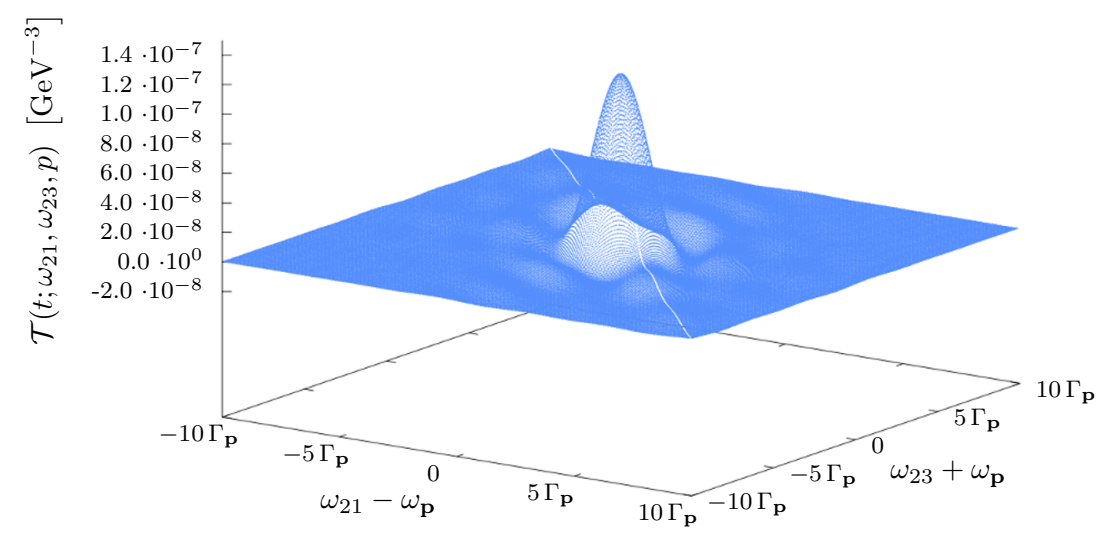

Figure 7. $\mathcal{T}\left(t ; \omega_{21}, \omega_{23}, p\right)$ around $\omega_{21}=-\omega_{23}=\omega_{\mathbf{p}}$ for fixed $t=10^{-2} / \mathrm{GeV}$ and $\omega_{\mathbf{p}}=1.42$. $10^{10} \mathrm{GeV}$ implying $\Gamma_{\mathbf{p}}=2.01 \cdot 10^{2} \mathrm{GeV}$ at $T=10^{11} \mathrm{GeV}, M=10^{10} \mathrm{GeV}$, and $\lambda^{2}=10^{-8}$.

2. $\omega_{21},-\omega_{23} \rightarrow \pm \omega_{\mathbf{p}}$ : symmetric under $\omega_{\mathbf{p}} \rightarrow-\omega_{\mathbf{p}}$,

$$
\begin{aligned}
& \lim _{-\omega_{23}, \omega_{21} \rightarrow \pm \omega_{\mathbf{p}}} \mathcal{T}\left(t ; \omega_{21}, \omega_{23}, p\right) \\
& =\frac{\mathrm{e}^{-\Gamma_{\mathbf{p}} t}}{\Gamma_{\mathbf{p}}^{3}\left(\Gamma_{\mathbf{p}}^{2}+16 \omega_{\mathbf{p}}^{2}\right)^{2}}\left[-8\left(\Gamma_{\mathbf{p}}^{4}+16 \Gamma_{\mathbf{p}}^{2} \omega_{\mathbf{p}}^{2}+128 \omega_{\mathbf{p}}^{4}\right)\right. \\
& +4 \mathrm{e}^{\Gamma_{\mathbf{p}} t}\left(\Gamma_{\mathbf{p}}^{4}\left(\Gamma_{\mathbf{p}} t-2\right)+8\left(3 \Gamma_{\mathbf{p}} t-4\right) \Gamma_{\mathbf{p}}^{2} \omega_{\mathbf{p}}^{2}+128\left(\Gamma_{\mathbf{p}} t-2\right) \omega_{\mathbf{p}}^{4}\right) \\
& -2 \mathrm{e}^{\Gamma_{\mathbf{p}} t / 2}\left(\left(\Gamma_{\mathbf{p}} t-4\right)\left(\Gamma_{\mathbf{p}}^{2}+16 \omega_{\mathbf{p}}^{2}\right)^{2}\right. \\
& \left.\left.+\Gamma_{\mathbf{p}}^{3}\left(\Gamma_{\mathbf{p}}\left(\Gamma_{\mathbf{p}} t-4\right)+16 \omega_{\mathbf{p}}^{2} t\right) \cos \left(2 \omega_{\mathbf{p}} t\right)\right)\right] \\
& \sim \mathcal{O}\left(\lambda^{-6} T^{-3}\right)
\end{aligned}
$$

Sufficiently far away from the peaks we can simplify $\mathcal{T}$ by considering $\Gamma_{\mathbf{p}} \rightarrow 0$, while keeping $\Gamma_{\mathbf{p}} t=$ const., which is possible because of the smallness of $\Gamma_{\mathbf{p}}$ [18],

$$
\begin{aligned}
\lim _{\Gamma_{\mathbf{p}} \rightarrow 0} \mathcal{T}\left(t ; \omega_{21}, \omega_{23}, p\right)= & \frac{\mathrm{e}^{-\Gamma_{\mathbf{p}} t / 2}}{\left(\omega_{21}+\omega_{23}\right)\left(\omega_{\mathbf{p}}^{2}-\omega_{23}^{2}\right)\left(\omega_{\mathbf{p}}^{2}-\omega_{21}^{2}\right)} \\
\times & {\left[\left(\omega_{21}-\omega_{23}\right) \omega_{\mathbf{p}} \sin \left(t \omega_{\mathbf{p}}\right)\left(\cos \left(t \omega_{21}\right)-\cos \left(t \omega_{23}\right)\right)\right.} \\
& +\left(\omega_{21} \omega_{23}-\omega_{\mathbf{p}}^{2}\right)\left(\cos \left(t \omega_{\mathbf{p}}\right)\left(\sin \left(t \omega_{21}\right)+\sin \left(t \omega_{23}\right)\right)\right. \\
& \left.\left.-\mathrm{e}^{\Gamma_{\mathbf{p}} t / 2} \sin \left(t\left(\omega_{21}+\omega_{23}\right)\right)\right)\right] .
\end{aligned}
$$

Since this is symmetric under the exchange of $\omega_{21}$ and $\omega_{23}$, we now consider $\left|\omega_{21}\right|>\left|\omega_{23}\right|$ without loss of generality. An upper bound is obtained by approximating $\left(\omega_{21}+\omega_{23}\right) \approx \omega_{21}$ in the denominator. We then have three cases of interest:

1. $\omega_{\mathbf{p}} \ll\left|\omega_{21}\right|,\left|\omega_{23}\right|$ : this is far away from the peaks and we have $\left(\omega_{\mathbf{p}}^{2}-\omega_{21}^{2}\right) \approx-\omega_{21}^{2}$, $\left(\omega_{\mathbf{p}}^{2}-\omega_{23}^{2}\right) \approx-\omega_{23}^{2}$, and $\left(\omega_{21} \omega_{23}-\omega_{\mathbf{p}}^{2}\right) \approx \omega_{21} \omega_{23}$.

$$
\mathcal{T}\left(t ; \omega_{21}, \omega_{23}, p\right) \sim \mathcal{O}\left(\omega_{21}^{-2} \omega_{23}^{-1}\right)
$$



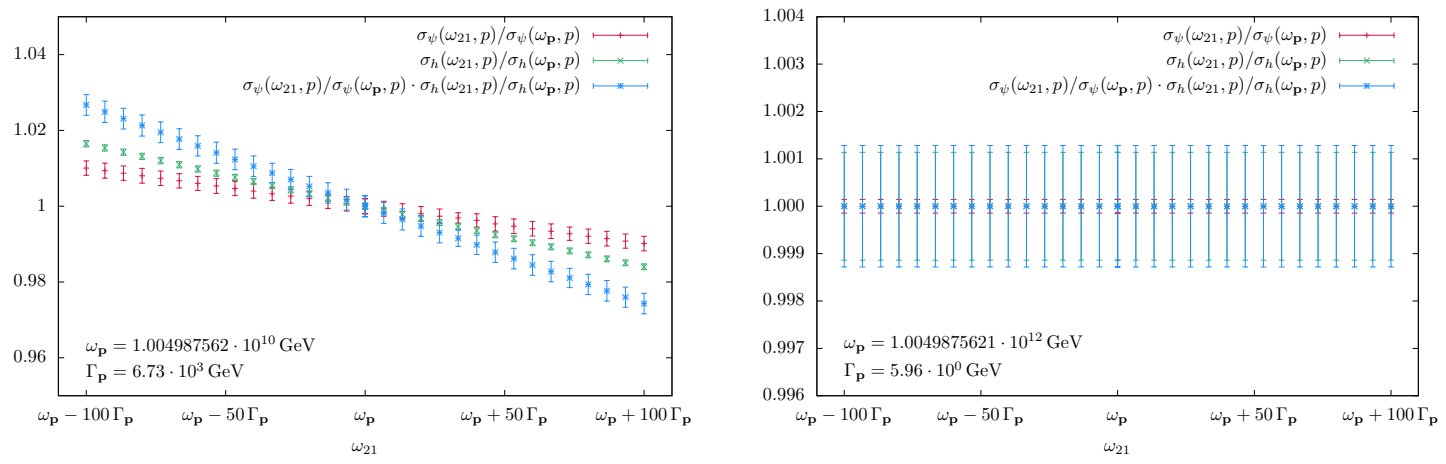

Figure 8. Deviation of the $\sigma_{h, \psi}\left(\omega_{21}, p\right)$ from the value at $\omega_{21}=\omega_{\mathbf{p}}$, plotted for $T=10^{11} \mathrm{GeV}$, $M=10^{10} \mathrm{GeV}$ and different $\omega_{\mathbf{p}}$.

2. $\omega_{21} \gg \omega_{\mathbf{p}}$ but $\omega_{23} \ll \omega_{\mathbf{p}}$ : in this case $\left(\omega_{\mathbf{p}}^{2}-\omega_{21}^{2}\right) \approx-\omega_{21}^{2},\left(\omega_{\mathbf{p}}^{2}-\omega_{23}^{2}\right) \approx \omega_{\mathbf{p}}^{2}$.

$$
\mathcal{T}\left(t ; \omega_{21}, \omega_{23}, p\right) \sim \mathcal{O}\left(\omega_{21}^{-2} T^{-1}\right)
$$

3. $\omega_{\mathbf{p}} \gg\left|\omega_{21}\right|,\left|\omega_{23}\right|:$ here we approximate $\left(\omega_{\mathbf{p}}^{2}-\omega_{21}^{2}\right) \approx \omega_{\mathbf{p}}^{2},\left(\omega_{\mathbf{p}}^{2}-\omega_{23}^{2}\right) \approx \omega_{\mathbf{p}}^{2}$, and $\left(\omega_{21} \omega_{23}-\omega_{\mathbf{p}}^{2}\right) \approx-\omega_{\mathbf{p}}^{2}$.

$$
\mathcal{T}\left(t ; \omega_{21}, \omega_{23}, p\right) \sim \mathcal{O}\left(\omega_{21}^{-1} T^{-2}\right)
$$

For very small values of $\left|\omega_{21}\right|$ we may expand the trigonometric functions in eq. (4.6), leading to

$$
\mathcal{T}\left(t ; \omega_{21}, \omega_{23}, p\right) \sim \frac{\Gamma_{\mathbf{p}} t\left(1-\mathrm{e}^{-\Gamma_{\mathbf{p}} t / 2} \cos \left(\omega_{\mathbf{p}} t\right)\right)}{\Gamma_{\mathbf{p}} \omega_{\mathbf{p}}^{2}},
$$

where we have kept $\Gamma_{\mathbf{p}}$ to compare with the peak region. For $\Gamma_{\mathbf{p}} t \sim \mathcal{O}(1)$ one finds

$$
\mathcal{T}\left(t ; \omega_{21}, \omega_{23}, p\right) \sim \mathcal{O}\left(\lambda^{-2} T^{-3}\right)
$$

Comparing all estimates shows that the region around the peak at $\omega_{21}=-\omega_{23}= \pm \omega_{\mathbf{p}}$ gives the largest contribution, $\mathcal{T}\left(t ; \omega_{21}, \omega_{23}, p\right) \sim \mathcal{O}\left(\lambda^{-6} T^{-3}\right)$. All other contributions are suppressed by a factor of order $\mathcal{O}\left(\lambda^{4}\right)$. Note that we later use $\lambda^{2}=10^{-8}$, which implies $\mathcal{O}\left(\lambda^{4}\right) \sim 10^{-16}$. This conclusion is corroborated by an independent consideration of the large time limit, which we give in appendix D. An exemplary plot of the function $\mathcal{T}\left(t ; \omega_{21}, \omega_{23}, p\right)$ illustrates our findings numerically in figure 7. 


\subsection{Approximate integration of the lepton number}

We are now going back to the full expression for the lepton number, eq. (3.36), and factor out the frequency integrals,

$$
\begin{aligned}
n_{L, i i}(t)= & -\frac{128}{\pi} \epsilon_{i i} \int_{0}^{t} \mathrm{~d} t_{1} \int_{0}^{t} \mathrm{~d} t_{2} \int_{0}^{t_{2}} \mathrm{~d} t_{3} \int_{0}^{\infty} \mathrm{d} p \frac{p^{2}}{\omega_{\mathbf{p}}} f_{F}\left(\omega_{\mathbf{p}}\right) \cos \left(\omega_{\mathbf{p}} \Delta t_{13}\right) \mathrm{e}^{-\Gamma_{\mathbf{p}} \frac{t_{13}}{2}} \\
& \times \mathcal{W}\left(t_{1}, t_{2}, t_{3} ; p\right) \\
\mathcal{W}\left(t_{1}, t_{2}, t_{3} ; p\right)= & \int_{-\infty}^{\infty} \frac{\mathrm{d} \omega_{21}}{2 \pi} \int_{-\infty}^{\infty} \frac{\mathrm{d} \omega_{23}}{2 \pi} f_{F}\left(\omega_{21}\right) \operatorname{Re}\left(\mathrm{e}^{-\mathrm{i}\left(\omega_{21} \Delta t_{21}+\omega_{23} \Delta t_{23}\right)}\right) \\
& \times\left[\sigma_{h}\left(\omega_{21}, p\right) \sigma_{h}\left(\omega_{23}, p\right)+\sigma_{\psi}\left(\omega_{21}, p\right) \sigma_{\psi}\left(\omega_{23}, p\right)\right]
\end{aligned}
$$

Following the analysis in the previous section, we restrict the frequency integrations to a diagonal strip including the on-shell peak, $\omega_{23} \in\left[-\omega_{21}-a,-\omega_{21}+a\right]$. We choose $a=$ const. $\times \Gamma_{\mathbf{p}}$, such that $\omega_{\mathbf{p}} \gg a \gg \Gamma_{\mathbf{p}}$ (cf. figure 7). Using the symmetry properties (3.25), we rewrite the $\omega_{21}$ integration to obtain

$$
\begin{aligned}
\mathcal{W}\left(t_{1}, t_{2}, t_{3} ; p\right) \simeq & \int_{0}^{\infty} \frac{\mathrm{d} \omega_{21}}{2 \pi} \int_{-\omega_{21}-a}^{-\omega_{21}+a} \frac{\mathrm{d} \omega_{23}}{2 \pi} \cos \left(\omega_{21} \Delta t_{21}+\omega_{23} \Delta t_{23}\right) \\
& \times\left[\sigma_{h}\left(\omega_{21}, p\right) \sigma_{h}\left(\omega_{23}, p\right)+\sigma_{\psi}\left(\omega_{21}, p\right) \sigma_{\psi}\left(\omega_{23}, p\right)\right] .
\end{aligned}
$$

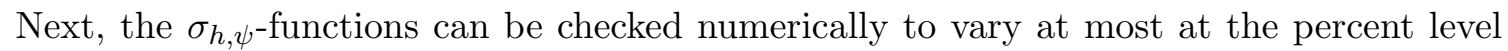
in the interval $\omega_{21},-\omega_{23} \in\left[\omega_{\mathbf{p}}-a, \omega_{\mathbf{p}}+a\right]$, as depicted in figure 8 , with the conservative choice $a=100 \Gamma_{\mathbf{p}}$. The variation is largest for small momenta $p$, which however do not contribute significantly to the integral. For the relevant momenta $p \sim \mathcal{O}(T)$, deviations from the on-shell values $\sigma_{h, \psi}\left(\omega_{21}=\omega_{\mathbf{p}}\right)$ are negligible. In a second step we may then only keep the leading term in a Taylor expansion around $\omega_{21}=-\omega_{23}=\omega_{\mathbf{p}}$ (cf. eq. (3.25)),

$$
\begin{array}{lll}
\sigma_{h}\left(\omega_{21}, p\right) \simeq \sigma_{h}\left(\omega_{\mathbf{p}}, p\right), & \sigma_{\psi}\left(\omega_{21}, p\right) \simeq \sigma_{\psi}\left(\omega_{\mathbf{p}}, p\right), \\
\sigma_{h}\left(\omega_{23}, p\right) \simeq \sigma_{h}\left(-\omega_{\mathbf{p}}, p\right)=\sigma_{\psi}\left(\omega_{\mathbf{p}}, p\right), & \sigma_{\psi}\left(\omega_{23}, p\right) \simeq \sigma_{\psi}\left(-\omega_{\mathbf{p}}, p\right)=\sigma_{h}\left(\omega_{\mathbf{p}}, p\right),
\end{array}
$$

allowing us to pull the $\sigma_{h, \psi}$ out of the integration. Note that it is sufficient to consider $+\omega_{\mathbf{p}}$ in this step, because we made use of the symmetry in $\omega_{21}$ before. We can now do the integration in eq. (4.13) analytically leading to

$$
\mathcal{W}\left(t_{1}, t_{2}, t_{3} ; p\right) \simeq \frac{\sin \left(a \Delta t_{23}\right)}{\pi \Delta t_{23}} \delta\left(\Delta t_{31}\right)\left[\sigma_{h}\left(\omega_{\mathbf{p}}, p\right) \sigma_{\psi}\left(\omega_{\mathbf{p}}, p\right)\right]
$$


Using this on-shell approximated intermediate result, we are able to carry out the time integration of the lepton number density

$$
\begin{aligned}
n_{L, i i}(t) \simeq & -\frac{128}{\pi} \epsilon_{i i} \int_{0}^{t} \mathrm{~d} t_{1} \int_{0}^{t} \mathrm{~d} t_{2} \int_{0}^{t_{2}} \mathrm{~d} t_{3} \int_{0}^{\infty} \mathrm{d} p \frac{p^{2}}{\omega_{\mathbf{p}}} f_{F}\left(\omega_{\mathbf{p}}\right)\left[\sigma_{h}\left(\omega_{\mathbf{p}}, p\right) \sigma_{\psi}\left(\omega_{\mathbf{p}}, p\right)\right] \\
& \times \cos \left(\omega_{\mathbf{p}} \Delta t_{13}\right) \mathrm{e}^{-\Gamma_{\mathbf{p}} \frac{t_{13}}{2}} \frac{\sin \left(a \Delta t_{23}\right)}{\pi \Delta t_{23}} \delta\left(\Delta t_{31}\right) \\
= & -\frac{128}{\pi} \epsilon_{i i} \int_{0}^{\infty} \mathrm{d} p \frac{p^{2}}{\omega_{\mathbf{p}}} f_{F}\left(\omega_{\mathbf{p}}\right)\left[\sigma_{h}\left(\omega_{\mathbf{p}}, p\right) \sigma_{\psi}\left(\omega_{\mathbf{p}}, p\right)\right] \frac{\mathrm{e}^{-\Gamma_{\mathbf{p}} t}}{4 \pi \Gamma_{\mathbf{p}}}\left[-\mathrm{i}\left(2 \operatorname{Ei}\left(t\left(-\mathrm{i} a+\Gamma_{\mathbf{p}}\right)\right)\right.\right. \\
& \left.\left.-2 \operatorname{Ei}\left(t\left(\mathrm{i} a+\Gamma_{\mathbf{p}}\right)\right)-\ln \left(\frac{-a-\mathrm{i} \Gamma_{\mathbf{p}}}{a-\mathrm{i} \Gamma_{\mathbf{p}}}\right)+\ln \left(\frac{-a+\mathrm{i} \Gamma_{\mathbf{p}}}{a+\mathrm{i} \Gamma_{\mathbf{p}}}\right)\right)+4 \mathrm{e}^{\Gamma_{\mathbf{p}} t} \operatorname{Si}(a t)\right],
\end{aligned}
$$

where $\operatorname{Ei}(x)$ denotes the exponential integral function and $\operatorname{Si}(x)$ the sine trigonometric integral function. Our choice $a=100 \Gamma_{\mathbf{p}}$ satisfies $a \gg \Gamma_{\mathbf{p}}$ and allows us to approximately consider $a t \rightarrow \infty$, while keeping $\Gamma_{\mathbf{p}} t$ fixed, leading to an $a$-independent result,

$$
n_{L, i i}(t) \simeq-\frac{64}{\pi} \epsilon_{i i} \int_{0}^{\infty} \mathrm{d} p \frac{p^{2}}{\omega_{\mathbf{p}}} f_{F}\left(\omega_{\mathbf{p}}\right) \frac{1-\mathrm{e}^{-\Gamma_{\mathbf{p}} t}}{\Gamma_{\mathbf{p}}} \sigma_{h}\left(\omega_{\mathbf{p}}, p\right) \sigma_{\psi}\left(\omega_{\mathbf{p}}, p\right) .
$$

Note that this expression only holds for $t \gtrsim 1 / \Gamma_{\mathbf{p}}$, since for earlier times the approximation leading to eq. (4.6) is not valid, i.e. the region around the on-shell peaks is less suppressed so that the peaks are less pronounced. These memory and off-shell effects could of course be kept by a complete numerical integration without restriction to the regions around $\omega_{21}=$ $-\omega_{23}= \pm \omega_{\mathbf{p}}$. Nevertheless, one makes the remarkable observation that at sufficiently late times our result has the same time dependence as the solution of the corresponding Boltzmann equations [18], but this time based on a full calculation without any assumptions.

Ultimately, we are interested in the value of the generated lepton number once it is thermalised. This is easily obtained by taking the limit $t \rightarrow \infty$, resulting in

$$
n_{L, i i}^{T} \simeq \lim _{t \rightarrow \infty} n_{L, i i}(t, t)=-\frac{64}{\pi} \epsilon_{i i} \int_{0}^{\infty} \mathrm{d} p \frac{p^{2}}{\omega_{\mathbf{p}}} f_{F}\left(\omega_{\mathbf{p}}\right) \frac{1}{\Gamma_{\mathbf{p}}}\left[\sigma_{h}\left(\omega_{\mathbf{p}}, p\right) \sigma_{\psi}\left(\omega_{\mathbf{p}}, p\right)\right] .
$$

As a valuable crosscheck for our approximate calculation, we change the order of integration in appendix $\mathrm{D}$, i.e. we first do the time integrals, followed by the $t \rightarrow \infty$ limit and only then integrate over the frequencies, arriving at the same result.

Finally, it is also interesting to quantify the timescale for thermalisation, which is expected to be of order $t_{T} \sim \mathcal{O}\left(1 / \Gamma_{\mathbf{p}}\right)$ according to the discussion in section 2.1. We define $t_{T}$ as the time where

$$
n_{L, i i}\left(t_{T}\right)=\left(1-\mathrm{e}^{-1}\right) n_{L, i i}^{T},
$$

which gives $t_{T}=1 / \Gamma$ for a constant $\Gamma_{\mathbf{p}} \equiv \Gamma$, cf. eq. (4.17). 

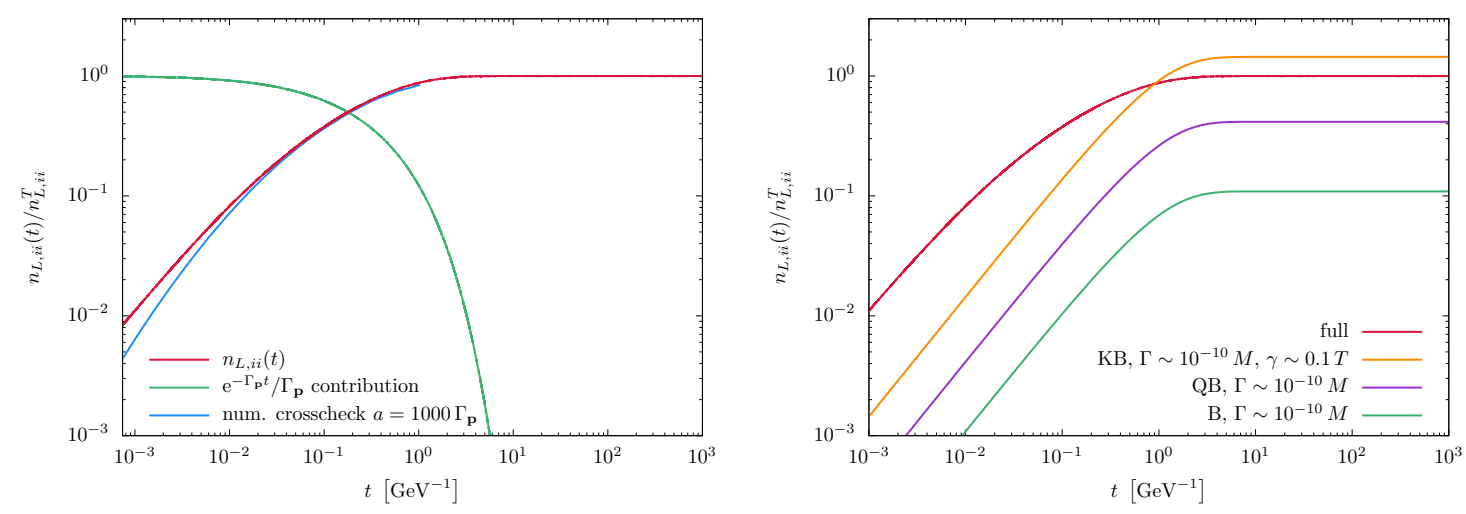

Figure 9. Time evolution of the lepton number density. Left: full result obtained by different orders of integration (see text). The green curve gives the time-dependent contribution. Right: comparison of the full result with Boltzmann (B), quantum Boltzmann (QB) and Kadanoff-Baym (KB) computations, where gauge corrections are not computed but parametrised by thermal widths.

\subsection{Time evolution of the lepton number}

The final integration over the Majorana neutrino momentum is performed numerically using integration and ODE algorithms implemented in the GNU scientific library [38] and the BOOST library [39]. Following [18], we consider a Majorana neutrino mass of $M=$ $10^{10} \mathrm{GeV}$ and a constant temperature of $T=10^{11} \mathrm{GeV}$ for the time-dependent calculation. This choice is consistent with the physical scenario outlined in section 2.1, allowing to neglect the Hubble expansion. For the coupling of the corrected Majorana neutrino decay width we choose $\lambda^{2}=10^{-8}$, in analogy to [20]. ${ }^{6}$ The SM couplings are obtained by solving the renormalization group equations from [40, 41] using the SM parameters from [3]. In analogy to the calculation of the asymptotic masses, we have neglected all contributions from the quark sector except for the top quark. All details are given in appendix E.

The resulting time evolution of the lepton number is shown in figure 9 (left) by the red curve. The blue curve corresponds to an alternative evaluation of the integrals, where we start from the time-integrated expression, $\mathcal{T}\left(t ; \omega_{21}, \omega_{23}, p\right)$ in appendix $\mathrm{C}$, and numerically integrate over the frequencies with $\omega_{23} \in\left[-\omega_{21}-a,-\omega_{21}+a\right]$, this time with the larger range $a=1000 \Gamma_{\mathbf{p}}$ and the full functions $\sigma_{h, \psi}$. As the figure shows, this crosscheck fully confirms the validity of our approximations at sufficiently large times. Deviations between the two curves show up only at early times, when the on-shell peak of the integrand is less pronounced. The green curve shows the time-dependent term in eq. (4.18), which dies away exponentially, leaving us with the fully thermalised value of the lepton number. The thermalisation time in the figure is consistent with the expectation for our choice of parameter values, i.e. we have $\Gamma_{\mathbf{p}} \sim \mathcal{O}(\mathrm{GeV})$ and $t_{T} \sim \mathcal{O}(1 / \mathrm{GeV})$.

In figure 9 (right) we compare our complete result to various other approaches discussed previously [18]. These are the solutions of Boltzmann equations (B), Boltzmann equations using quantum mechanical distribution functions (QB), and Kadanoff-Baym equations without gauge corrections, but with thermal widths for the lepton and Higgs propaga-

\footnotetext{
${ }^{6}$ In fact our presentation is independent of the choice of $\lambda^{2}$ because it is possible to rescale $t$ in eq. (4.17) accordingly and factor $\lambda^{2}$ out of $1 / \Gamma_{\mathbf{p}}$ together with $\lambda_{i i}$.
} 

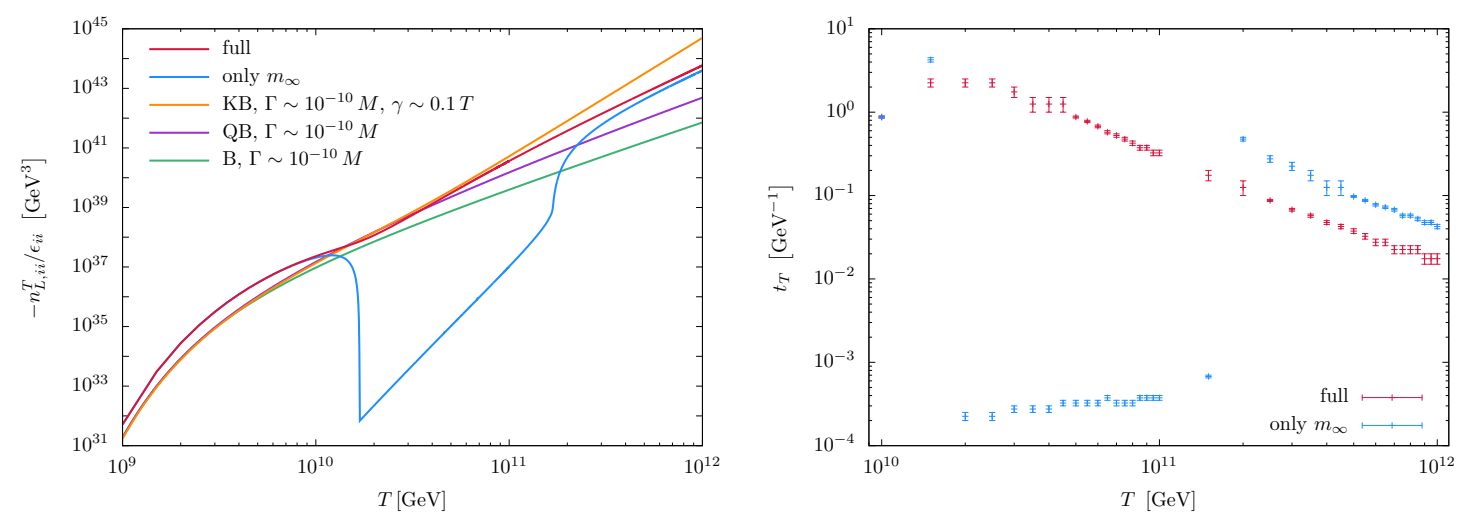

Figure 10. Left: thermalised lepton number density from the full resummation, only including asymptotic masses, Boltzmann (B), quantum Boltzmann (QB) and Kadanoff-Baym with parametrised propagators $(\mathrm{KB})$, as a function of temperature. Right: thermalisation time as a function of temperature.

tors, $\gamma=\gamma_{l}+\gamma_{\phi}$, introduced by hand (KB). ${ }^{7}$ In all three cases we work with a constant decay width for the Majorana neutrino propagator, $\Gamma_{\mathbf{p}}\left(\omega_{\mathbf{p}}\right)=\Gamma \sim 10^{-10} M \sim \mathcal{O}(\mathrm{GeV})$, corresponding to the dominant contribution in our full calculation. For the SM thermal damping widths we choose $\gamma \sim 0.1 T$ as expected from thermal field theory [29]. All results are normalised by the value of the thermalised lepton number density from the full calculation. While all curves have the same qualitative features, the Boltzmann result deviates by almost an order of magnitude, the quantum Boltzmann computation reduces the difference significantly and Kadanoff-Baym with thermal widths is $\mathcal{O}(1)$ accurate. The qualitative similarity is due to eq. (4.17) having the same explicit time dependence as the Boltzmann solutions. The quantitative difference of the full solution stems from the functions $\sigma_{h, \psi}$ and a momentum-dependent $\Gamma_{\mathbf{p}}$, effecting different changes for the weights of the momentum modes. We may thus conclude that gauge corrections indeed cause the expected narrow damping widths at late times. However, as expected in $[18,19]$, for quantitative results the full calculation is mandatory.

\subsection{Temperature dependence of the gauge corrections}

In the simplest leptogenesis scenario considered here, the temperature is constant to first approximation. However, gauge corrections apply to other parameter choices and scenarios as well, and it is interesting to investigate their relative importance as a function of temperature. In figure 10 (left) we repeat the comparison of the previous section in case of the thermalised lepton number as a function of temperature $T$. Only including asymptotic masses leads to a kinematical suppression of the Majorana decay and production rate [20], and hence of the lepton number, in some temperature range, which is unphysical. This illustrates the importance of a complete calculation including the resummed gauge corrections. At yet higher temperatures, once $T \gg M$, the kinematic suppression is switched off and only including asymptotic masses gives a good approximation of the full result.

\footnotetext{
${ }^{7}$ To be specific, we use eq. (7.2) from [18] and perform an additional k-integration, inserting eq. (7.3) for (B), eq. (7.10) for (QB) and eq. (7.8) for (KB).
} 
For low temperatures $T \lesssim M$, the results of Boltzmann, quantum Boltzmann and Kadanoff-Baym with thermal damping widths are very close. This agrees with the smallness of corrections observed in the non-relativistic case [23]. Since the resummed gauge corrections are suppressed in this regime, our calculation is close to the previous results as well. Note however, that the agreement cannot be exact, because the weights of the momentum modes are still not identical, due to the momentum dependent $\Gamma_{\mathbf{p}}$. It is thus the choice of the (momentum independent) model parameters $\Gamma$ and $\gamma$ which determines the temperature range of best agreement between the gauge resummed and approximate solutions. For the choice presented here this is at $T \sim M$. Another choice achieves full agreement at low temperatures at the expense of larger discrepancies at high temperatures, while an appropriately temperature dependent $\Gamma$ can increase the range of agreement.

For temperatures $T>M$, the different solutions split up. Introducing thermal damping widths by hand repairs the qualitative deficit of kinematic suppression (and agrees well with the full result in a part of this region), but overestimates the lepton number for high temperatures. This is because the dominant momenta become more light-like, and hence the resummation more important. Note however that, by altering the scaling of the Majorana neutrino decay width to e.g. $\Gamma \sim 10^{-11} T$, better agreement with the full result is achieved.

Finally, figure 10 (right) shows the thermalisation times for the lepton number, extracted from the full result and the one including asymptotic masses only. Apart from the region with kinematic suppression we roughly find a scaling $t_{T} \sim \mathcal{O}\left(1 / \Gamma_{\mathbf{p}}\right) \propto 1 / T$ as expected.

\section{Conclusions}

In this work the leading-order gauge corrections to quantum leptogenesis were included in a fully quantum field theoretical calculation based on non-equilibrium Kadanoff-Baym equations. In this setting, gauge corrections appear first at the three-loop level of lepton self-energies. In the symmetric electroweak phase, gauge bosons are massless on tree-level and, for $T \gtrsim M$ and collinear thermal loop momenta, require resummations to infinite loop order to complete a leading-order calculation in the gauge coupling. We have adapted a previous CTL resummation of the Majorana neutrino self-energy in such a way that it can be applied to the lepton self-energy graphs relevant for quantum leptogenesis. Our resulting lepton number density, eq. (3.36), is complete to leading order in all SM couplings and forms the basis for a quantitatively accurate evaluation of leptogenesis.

We have evaluated our expression for the simplest vanilla leptogenesis scenario at late times approaching thermalisation, which allows for an analytic analysis and integration of the lepton number density in Fourier space. One observes how the gauge corrections dynamically cause a dominant peak to grow in the integrand, whose width at late times corresponds to the thermal decay width of the Majorana neutrino. Similarly, thermal widths for leptons and Higgs propagators are automatically and fully included. The resulting late time behaviour qualitatively agrees with that observed from Boltzmann equations. However, for quantitative results, the full quantum calculation is necessary. Finally, we have 
evaluated the temperature dependence of the gauge corrections and the thermalisation time to estimate effects on other parameter choices or scenarios. Boltzmann equations dressed with quantum distribution functions underestimate the full result by $\mathcal{O}(1)$ for $T \gtrsim M$, introducing an uncertainty of similar size as that of spectator processes. Thus, we have also obtained a first complete estimate of the theoretical uncertainties for leptogenesis. On the other hand, for $T \lesssim M$ the resummed gauge corrections are suppressed and our result is close to the previous ones.

Our calculation can be easily adopted to other parameter regions or modfied scenarios, such as resonant leptogenesis. For yet better accuracy, the calculation could be further refined by also including washout diagrams as well as the temperature change due to the Hubble expansion.

\section{Acknowledgments}

We thank D. Bödeker and W. Buchmüller for useful discussions and suggestions. Numerical calculations were performed on the LOEWE-CSC computer at Goethe University Frankfurt. This work is supported by the ERC Starting Grant 'NewAve' (638528) as well as by the Deutsche Forschungsgemeinschaft under Germany's Excellence Strategy — EXC 2121 'Quantum Universe' - 390833306.

\section{A Feynman diagrams for gauge corrections at three-loop level}

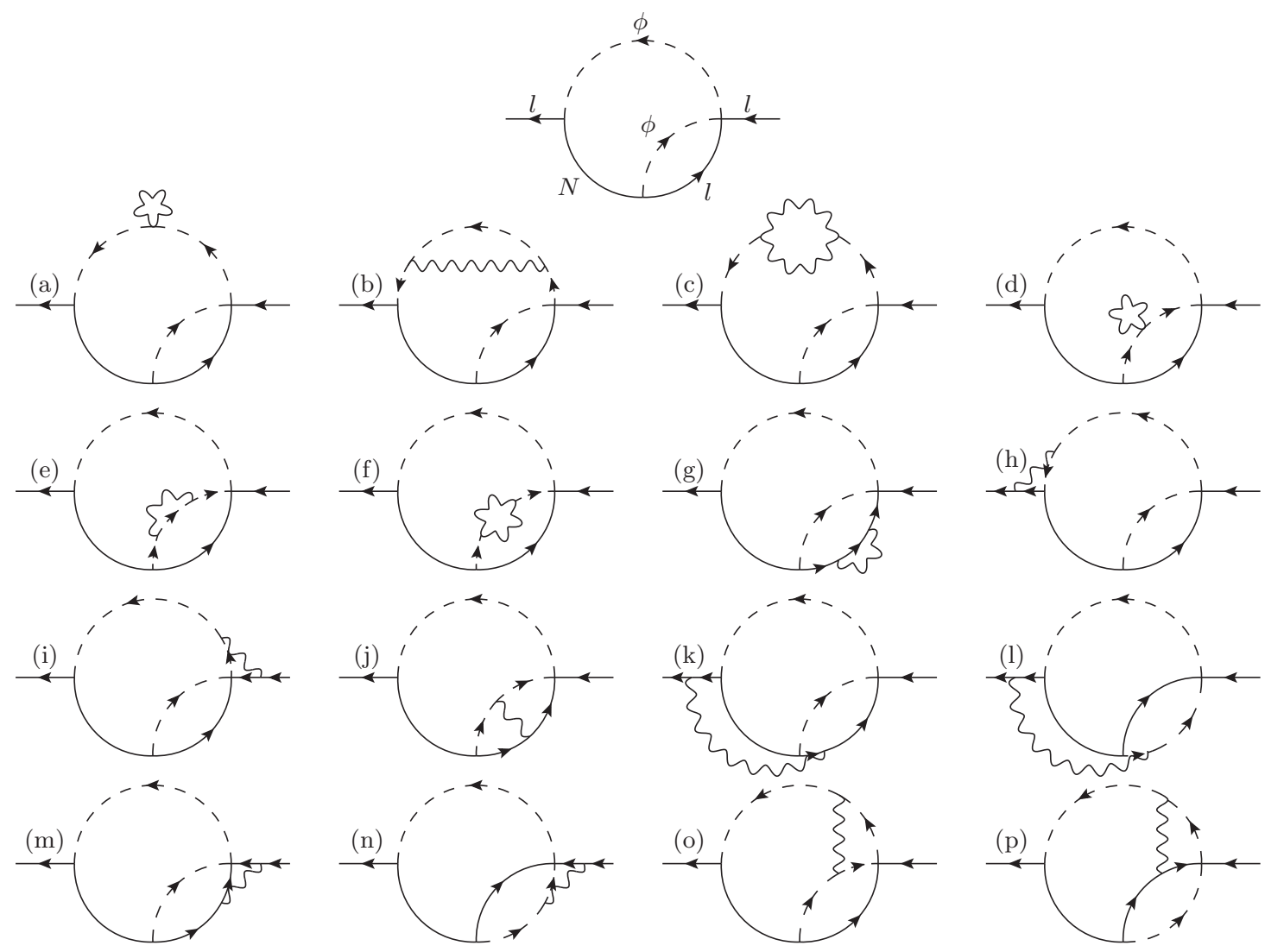


Here we list all Feynman diagrams obtained by adding one gauge boson line to the diagrams in figure 3. The diagrams (a)-(g) are gauge corrections to the lepton and Higgs propagators, which are included by the asymptotic masses, eq. (3.1). The diagrams (h)-(j) are vertex gauge corrections, which are included in the resummed ladder diagram figure 6 . The remaining diagrams are further suppressed because the mean free path between heavy neutrino vertices exceeds the Debye screening length, for details see appendix B.

\section{B Leading and sub-leading gauge corrections}

In order to distinguish leading and sub-leading gauge corrections, as well as for generalisations to other neutrino mass configurations, it is useful to step back from the hierarchical scenario and re-introduce the heavy neutrinos $N_{2,3}$. The self-energy diagrams of the neutrino $N$ can then be separated into two classes, which can be described as either "propagator-type" or "vertex-type" corrections, figures 11 and 12, respectively.

A parametric suppression applies to a diagram whenever the mean free path between emission and absorbtion of a soft gauge boson exceeds its associated screening length [36]. In our case, the mean free path of the heavy neutrinos is $l_{N_{i}} \sim\left(\lambda^{2} M_{i}\right)^{-1}$, while the Debye screening length is $l_{D} \sim(g T)^{-1}$. We thus have $l_{N} \gg l_{D}$ as long as

$$
M_{i} \ll \frac{g}{\lambda^{2}} T
$$

With $\lambda^{2} \sim 10^{-8}$, this is satisfied for all mass scenarios provided the $M_{i}$ are sufficiently below the Planck scale. Hence, the diagrams (k)-(n) in figure 11 and (i), (j), (m), (n) in figure 12 are parametrically suppressed. A similar suppression occurs for vertex corrections as (k), (l) in figure 12, once the $N$-line is closed. All remaining diagrams have to be resummed, as explained in section 3.1. Finally, we return to the hierarchical scenario and integrate out the $N_{2,3}$, where both propagator-type and vertex-type diagrams reduce to a double blob diagram with an effective four-point vertex. Upon closing the external lines we then obtain the resummed diagram in figure 6 . 

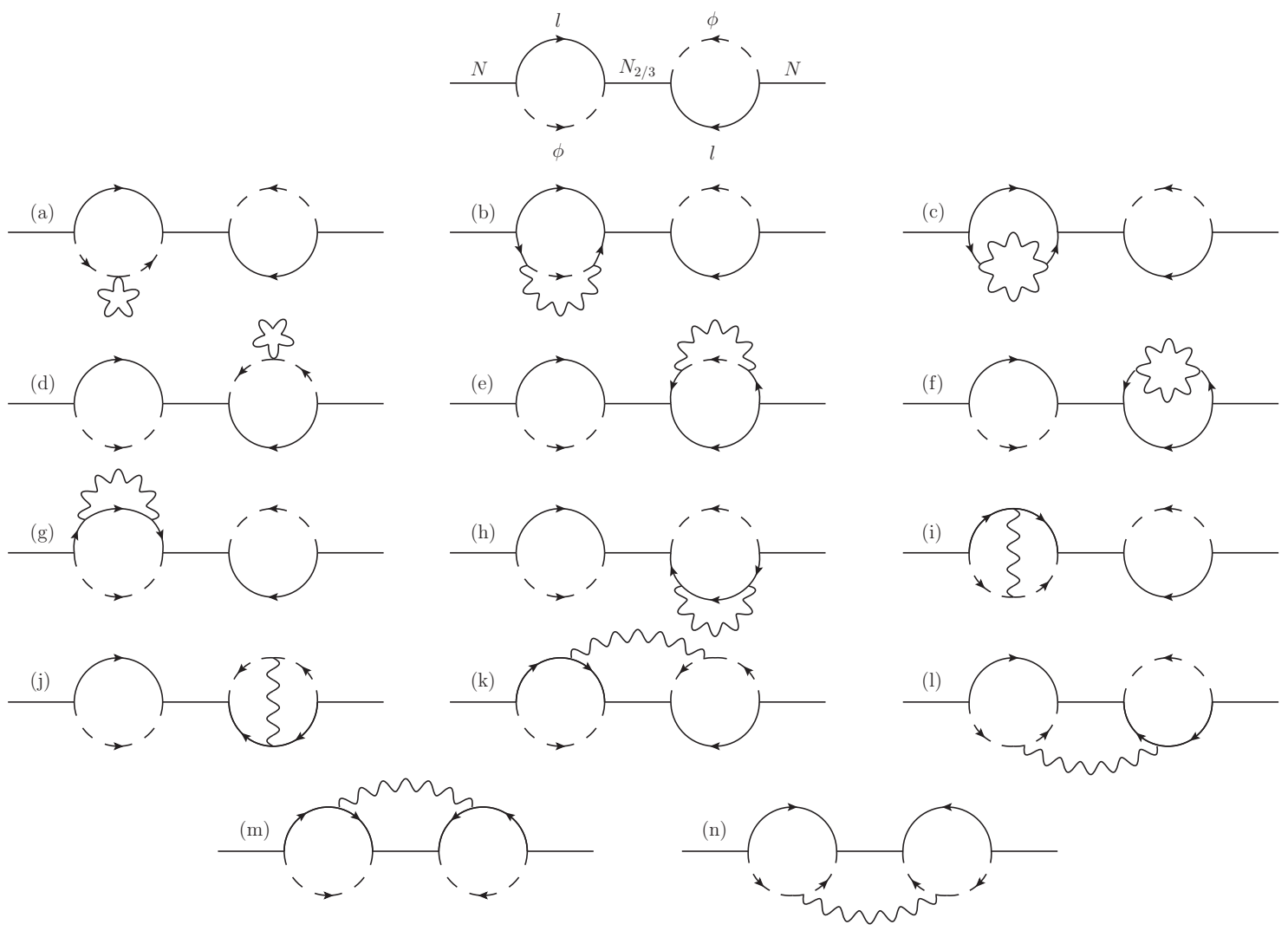

Figure 11. Gauge corrections to the propagator-type self-energy contribution of $N$.

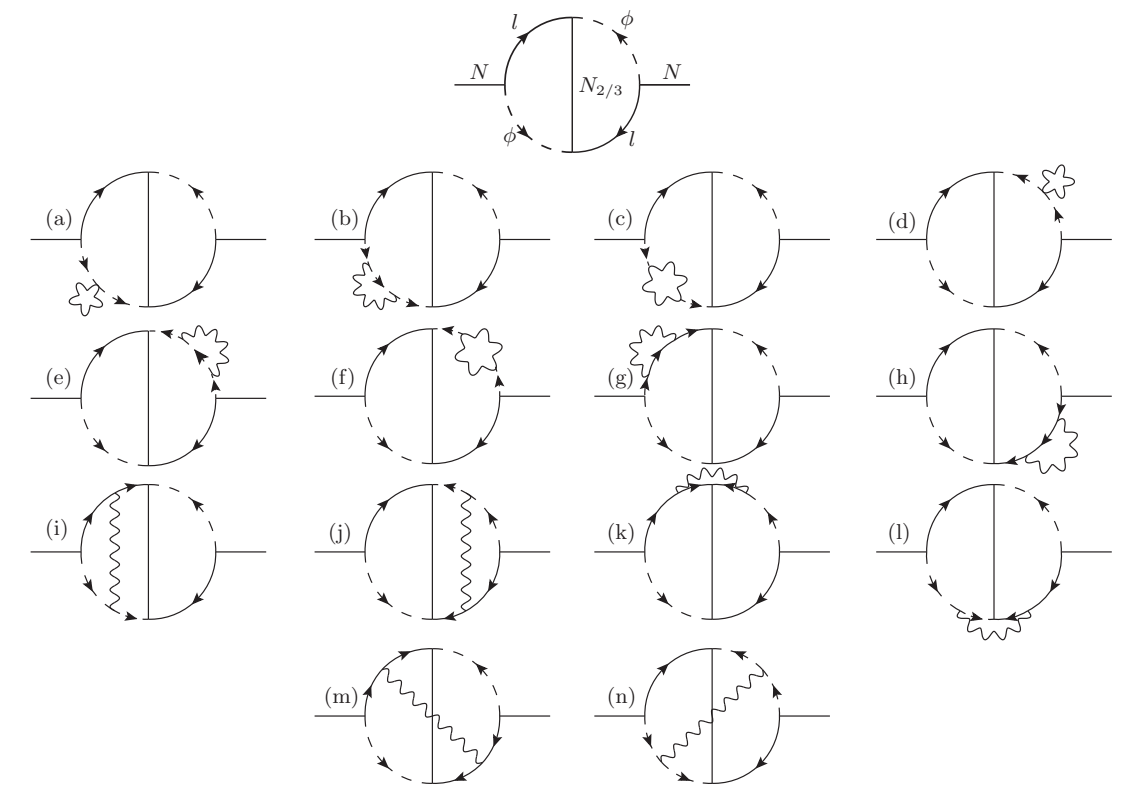

Figure 12. Gauge corrections to the vertex-type self-energy contribution of $N$. 


\section{The function $\mathcal{T}\left(t ; \omega_{21}, \omega_{23}, p\right)$}

The integral in eq. (4.2) can be performed analytically giving the result

$$
\begin{aligned}
& \mathcal{T}\left(t ; \omega_{21}, \omega_{23}, p\right)=\frac{\mathrm{e}^{-\Gamma_{\mathbf{p}} t}}{\left(\omega_{21}+\omega_{23}\right)\left(\Gamma_{\mathbf{p}}^{2}+4\left(\omega_{23}-\omega_{\mathbf{p}}\right)^{2}\right)\left(\Gamma_{\mathbf{p}}^{2}+4\left(\omega_{23}+\omega_{\mathbf{p}}\right)^{2}\right)} \\
& \times \frac{1}{\left(\Gamma_{\mathbf{p}}^{2}+4\left(\omega_{21}-\omega_{\mathbf{p}}\right)^{2}\right)\left(\Gamma_{\mathbf{p}}^{2}+4\left(\omega_{21}+\omega_{\mathbf{p}}\right)^{2}\right)}\left\{8 \Gamma_{\mathbf{p}}\left(\omega_{21}+\omega_{23}\right)\right. \\
& \times\left[-\left(\Gamma_{\mathbf{p}}^{2}+4 \omega_{21}^{2}\right)\left(\Gamma_{\mathbf{p}}^{2}+4 \omega_{23}^{2}\right)-8\left(\Gamma_{\mathbf{p}}^{2}+2\left(\omega_{21}^{2}-4 \omega_{21} \omega_{23}+\omega_{23}^{2}\right)\right) \omega_{\mathbf{p}}^{2}-16 \omega_{\mathbf{p}}^{4}\right] \\
& -8 \mathrm{e}^{\Gamma_{\mathbf{p}} t} \Gamma_{\mathbf{p}}\left(\omega_{21}+\omega_{23}\right)\left[\left(\Gamma_{\mathbf{p}}^{2}+4 \omega_{21}^{2}\right)\left(\Gamma_{\mathbf{p}}^{2}+4 \omega_{23}^{2}\right)+8\left(\Gamma_{\mathbf{p}}^{2}+2\left(\omega_{21}^{2}-4 \omega_{21} \omega_{23}+\omega_{23}^{2}\right)\right) \omega_{\mathbf{p}}^{2}+16 \omega_{\mathbf{p}}^{4}\right] \\
& \times \cos \left(t\left(\omega_{21}+\omega_{23}\right)\right)+4 \mathrm{e}^{\Gamma_{\mathbf{p}} t}\left(\Gamma_{\mathbf{p}}^{2}-4 \omega_{21} \omega_{23}+4 \omega_{\mathbf{p}}^{2}\right) \sin \left(t\left(\omega_{21}+\omega_{23}\right)\right) \\
& \times\left[\Gamma_{\mathbf{p}}^{4}+16\left(\omega_{21}-\omega_{\mathbf{p}}\right)\left(\omega_{23}-\omega_{\mathbf{p}}\right)\left(\omega_{21}+\omega_{\mathbf{p}}\right)\left(\omega_{23}+\omega_{\mathbf{p}}\right)+4 \Gamma_{\mathbf{p}}^{2}\left(\omega_{21}^{2}+\omega_{23}^{2}+2 \omega_{\mathbf{p}}^{2}\right)\right] \\
& +8 \mathrm{e}^{\Gamma_{\mathbf{p}} t / 2}\left[\operatorname { c o s } ( t ( \omega _ { 2 1 } - \omega _ { 2 3 } ) / 2 ) \operatorname { c o s } ( t \omega _ { \mathbf { p } } ) \left(2 \Gamma _ { \mathbf { p } } ( \omega _ { 2 1 } + \omega _ { 2 3 } ) \left(\left(\Gamma_{\mathbf{p}}^{2}+4 \omega_{21}^{2}\right)\left(\Gamma_{\mathbf{p}}^{2}+4 \omega_{23}^{2}\right)\right.\right.\right. \\
& \left.+8\left(\Gamma_{\mathbf{p}}^{2}+2\left(\omega_{21}^{2}-4 \omega_{21} \omega_{23}+\omega_{23}^{2}\right)\right) \omega_{\mathbf{p}}^{2}+16 \omega_{\mathbf{p}}^{4}\right) \cos \left(t\left(\omega_{21}+\omega_{23}\right) / 2\right) \\
& -\left(\Gamma_{\mathbf{p}}^{4}+16\left(\omega_{21}-\omega_{\mathbf{p}}\right)\left(\omega_{23}-\omega_{\mathbf{p}}\right)\left(\omega_{21}+\omega_{\mathbf{p}}\right)\left(\omega_{23}+\omega_{\mathbf{p}}\right)+4 \Gamma_{\mathbf{p}}^{2}\left(\omega_{21}^{2}+\omega_{23}^{2}+2 \omega_{\mathbf{p}}^{2}\right)\right) \\
& \left.\times\left(\Gamma_{\mathbf{p}}^{2}-4 \omega_{21} \omega_{23}+4 \omega_{\mathbf{p}}^{2}\right) \sin \left(1 / 2 t\left(\omega_{21}+\omega_{23}\right)\right)\right)-4\left(\omega_{21}-\omega_{23}\right) \omega_{\mathbf{p}} \sin \left(t\left(\omega_{21}-\omega_{23}\right) / 2\right) \\
& \times\left(-4 \Gamma_{\mathbf{p}}\left(\omega_{21}+\omega_{23}\right)\left(\Gamma_{\mathbf{p}}^{2}-4 \omega_{21} \omega_{23}+4 \omega_{\mathbf{p}}^{2}\right) \cos \left(t\left(\omega_{21}+\omega_{23}\right) / 2\right)\right. \\
& +\left(\Gamma_{\mathbf{p}}^{4}+16\left(\omega_{21}-\omega_{\mathbf{p}}\right)\left(\omega_{23}-\omega_{\mathbf{p}}\right)\left(\omega_{21}+\omega_{\mathbf{p}}\right)\left(\omega_{23}+\omega_{\mathbf{p}}\right)-4 \Gamma_{\mathbf{p}}^{2}\left(\omega_{21}^{2}+4 \omega_{21} \omega_{23}+\omega_{23}^{2}-2 \omega_{\mathbf{p}}^{2}\right)\right) \\
& \left.\left.\left.\times \sin \left(1 / 2 t\left(\omega_{21}+\omega_{23}\right)\right)\right) \sin \left(t \omega_{\mathbf{p}}\right)\right]\right\} .
\end{aligned}
$$

\section{Late-time limit for the lepton number}

Here we provide a crosscheck for the derivation of the approximated lepton number density in the infinite-time limit. Starting from the full expression, eq. (4.1), taking the infinite time limit in the time dependent part $\mathcal{T}\left(t ; \omega_{21}, \omega_{23}, p\right)$ of the integrand reads

$$
\lim _{t \rightarrow \infty} \mathcal{T}\left(t ; \omega_{21}, \omega_{23}, p\right)=\frac{4 \pi\left(\Gamma_{\mathbf{p}}^{2}+4\left(\omega_{\mathbf{p}}^{2}+\omega_{21}^{2}\right)\right)}{\Gamma_{\mathbf{p}}^{4}+16\left(\omega_{\mathbf{p}}^{2}-\omega_{21}^{2}\right)^{2}+8 \Gamma_{\mathbf{p}}^{2}\left(\omega_{\mathbf{p}}^{2}+\omega_{21}^{2}\right)} \delta\left(\omega_{21}+\omega_{23}\right) .
$$

Note the explicit appearance of a Delta function enforcing $\omega_{23}=-\omega_{21}$, which is consistent with the dominant on-shell approximation $\omega_{21}= \pm \omega_{\mathbf{p}}, \omega_{23}=\mp \omega_{\mathbf{p}}$ identified in section 4.1.

Carrying out the $\omega_{23}$ integration in the infinite-time limit and using the symmetry properties of the $\sigma$-functions (3.25), we arrive at

$$
\begin{aligned}
\lim _{t \rightarrow \infty} n_{L, i i}(t)= & -\frac{128}{\pi^{2}} \epsilon_{i i} \int_{0}^{\infty} d p \int_{-\infty}^{\infty} \frac{d \omega_{21}}{2 \pi} \frac{p^{2}}{\omega_{\mathbf{p}}} \frac{4 \pi f_{F}\left(\omega_{\mathbf{p}}\right)\left(\Gamma_{\mathbf{p}}^{2}+4\left(\omega_{\mathbf{p}}^{2}+\omega_{21}^{2}\right)\right)}{\Gamma_{\mathbf{p}}^{4}+16\left(\omega_{\mathbf{p}}^{2}-\omega_{21}^{2}\right)^{2}+8 \Gamma_{\mathbf{p}}^{2}\left(\omega_{\mathbf{p}}^{2}+\omega_{21}^{2}\right)} \\
& \times \sigma_{\psi}\left(\omega_{21}, p\right) \sigma_{h}\left(\omega_{21}, p\right) .
\end{aligned}
$$


Approximating the $\sigma_{h, \psi}$-functions by their on-shell contributions, cf. section 4.2,

$$
\sigma_{\psi}\left(\omega_{21}, p\right) \approx \sigma_{\psi}\left(\omega_{\mathbf{p}}, p\right), \quad \sigma_{h}\left(\omega_{21}, p\right) \approx \sigma_{h}\left(\omega_{\mathbf{p}}, p\right)
$$

we can do the $\omega_{21}$-integral to recover

$$
\lim _{t \rightarrow \infty} n_{L, i i}(t)=-\frac{64}{\pi} \epsilon_{i i} \int_{0}^{\infty} d p \frac{p^{2}}{\omega_{\mathbf{p}}} f_{F}\left(\omega_{\mathbf{p}}\right) \frac{1}{\Gamma_{\mathbf{p}}} \sigma_{\psi}\left(\omega_{\mathbf{p}}, p\right) \sigma_{h}\left(\omega_{\mathbf{p}}, p\right),
$$

as in eq. (4.18), validating our approximate integration in section 4.2.

\section{E SM parameters for the numerical evaluation}

In order to evolve the SM parameters to the scale given by the temperature $\mu=2 \pi T$, we employ the renormalization group equations (RGEs) from [40, 41] taking only into account the largest coupling to a quark, i.e. the top quark being the heaviest quark,

$$
\begin{aligned}
& \frac{\mathrm{d} g^{\prime 2}}{\mathrm{~d} \tau}=\frac{g^{\prime 4}}{8 \pi^{2}} \frac{41}{10}+\mathcal{O}\left(g_{\mathrm{SM}}^{6}\right), \frac{g^{4}}{\mathrm{~d} g^{2}}=\frac{19}{8 \pi^{2}}\left(-\frac{19}{6}\right)+\mathcal{O}\left(g_{\mathrm{SM}}^{6}\right), \\
& \frac{\mathrm{d} g_{s}^{2}}{\mathrm{~d} \tau}=\frac{g_{s}^{4}}{8 \pi^{2}}(-7)+\mathcal{O}\left(g_{\mathrm{SM}}^{6}\right), \\
& \frac{\mathrm{d} h_{t}^{2}}{\mathrm{~d} \tau}=\frac{h_{t}^{2}}{8 \pi^{2}}\left(\frac{9}{2} h_{t}^{2}-\frac{17}{20} g^{\prime 2}-\frac{9}{4} g^{2}-8 g_{s}^{2}\right)+\mathcal{O}\left(g_{\mathrm{SM}}^{6}\right), \\
& \frac{\mathrm{d} \lambda_{\phi}}{\mathrm{d} \tau}=\frac{1}{16 \pi^{2}}\left(\frac{27}{200} g^{\prime 4}+\frac{9}{20} g^{\prime 2} g^{2}+\frac{9}{8} g^{4}-\frac{9}{5} g^{\prime 2} \lambda_{\phi}-9 g^{2} \lambda_{\phi}\right. \\
&\left.\quad-6 h_{t}^{4}+12 h_{t}^{2} \lambda_{\phi}+24 \lambda_{\phi}^{2}\right)+\mathcal{O}\left(g_{\mathrm{SM}}^{6}\right),
\end{aligned}
$$

where $\tau \equiv \ln \left(\mu / \mu_{0}\right)$ and we choose $\mu_{0}=2 \pi T_{0}$ with $T_{0}=10^{9} \mathrm{GeV}$. For notational simplicity $\mathcal{O}\left(g_{\mathrm{SM}}^{6}\right)$ denotes any combination of SM couplings and $\lambda_{\phi} \sim \mathcal{O}\left(g_{\mathrm{SM}}^{2}\right)$ for this counting. As initial conditions we use at $\tau_{Z} \equiv \ln \left(m_{Z} / \mu_{0}\right)[3]$

$$
\begin{aligned}
m_{Z} & =91.1876(21) \mathrm{GeV} \\
\alpha_{e m}\left(\tau_{Z}\right) & =1 / 127.950(17) \\
\alpha_{s}\left(\tau_{Z}\right) & =0.1182(16) \\
\sin ^{2} \theta_{W}\left(\tau_{Z}\right) & =0.23129(5) \\
m_{t} & =173.21(1.22) \mathrm{GeV} \\
m_{H} & =125.09(24) \mathrm{GeV} \\
G_{F} & =1.1663787(6) \cdot 10^{-5} \mathrm{GeV}^{-2} \Rightarrow v=\frac{1}{\sqrt{\sqrt{2} G_{F}}}=246.2197(1) \mathrm{GeV}
\end{aligned}
$$


With the relations between the couplings given in [40], one analytically obtains

$$
\begin{aligned}
& g^{\prime 2}(\tau)=\frac{c_{1}}{d_{1}-\tau}, \quad c_{1}=\frac{80 \pi^{2}}{41}, \quad d_{1}=\frac{20 \pi\left(1-\sin ^{2} \theta_{W}\left(\tau_{Z}\right)\right)}{41 \alpha_{e m}\left(\tau_{Z}\right)}+\tau_{Z}, \\
& g^{2}(\tau)=\frac{c_{2}}{d_{2}+\tau}, \quad c_{2}=\frac{48 \pi^{2}}{19}, \quad d_{2}=\frac{12 \pi \sin ^{2} \theta_{W}\left(\tau_{Z}\right)}{19 \alpha_{e m}\left(\tau_{Z}\right)}-\tau_{Z}, \\
& g_{s}^{2}(\tau)=\frac{c_{3}}{d_{3}+\tau}, \quad c_{3}=\frac{8 \pi^{2}}{7}, \quad d_{3}=\frac{2 \pi}{7 \alpha_{s}\left(\tau_{Z}\right)}-\tau_{Z},
\end{aligned}
$$

which we then use to solve the RGEs for $h_{t}$ and $\lambda_{\phi}$ numerically.

Open Access. This article is distributed under the terms of the Creative Commons Attribution License (CC-BY 4.0), which permits any use, distribution and reproduction in any medium, provided the original author(s) and source are credited.

\section{References}

[1] V.A. Kuzmin, V.A. Rubakov and M.E. Shaposhnikov, On the anomalous electroweak baryon number nonconservation in the early universe, Phys. Lett. B 155 (1985) 36 [INSPIRE].

[2] P. Huet and E. Sather, Electroweak baryogenesis and standard model CP-violation, Phys. Rev. D 51 (1995) 379 [hep-ph/9404302] [INSPIRE].

[3] Particle Data Group collaboration, Review of particle physics, Chin. Phys. C 40 (2016) 100001 [INSPIRE].

[4] W. Buchmüller and O. Philipsen, Phase structure and phase transition of the $\mathrm{SU}(2)$ Higgs model in three-dimensions, Nucl. Phys. B 443 (1995) 47 [hep-ph/9411334] [INSPIRE].

[5] K. Kajantie, M. Laine, K. Rummukainen and M.E. Shaposhnikov, The electroweak phase transition: a nonperturbative analysis, Nucl. Phys. B 466 (1996) 189 [hep-lat/9510020] [INSPIRE].

[6] F. Csikor, Z. Fodor and J. Heitger, Where does the hot electroweak phase transition end?, Nucl. Phys. B Proc. Suppl. 73 (1999) 659 [hep-ph/9809293] [InSPIRE].

[7] M. Fukugita and T. Yanagida, Baryogenesis without grand unification, Phys. Lett. B 174 (1986) 45 [INSPIRE].

[8] S. Blanchet and P. Di Bari, The minimal scenario of leptogenesis, New J. Phys. 14 (2012) 125012 [arXiv: 1211.0512] [INSPIRE].

[9] G. 't Hooft, Symmetry breaking through Bell-Jackiw anomalies, Phys. Rev. Lett. 37 (1976) 8 [INSPIRE].

[10] G. 't Hooft, Computation of the quantum effects due to a four-dimensional pseudoparticle, Phys. Rev. D 14 (1976) 3432 [Erratum ibid. 18 (1978) 2199] [INSPIRE].

[11] R.N. Mohapatra and G. Senjanović, Neutrino mass and spontaneous parity nonconservation, Phys. Rev. Lett. 44 (1980) 912 [INSPIRE].

[12] S. Biondini et al., Status of rates and rate equations for thermal leptogenesis, Int. J. Mod. Phys. A 33 (2018) 1842004 [arXiv:1711.02864] [INSPIRE].

[13] M. Drewes et al., ARS leptogenesis, Int. J. Mod. Phys. A 33 (2018) 1842002 [arXiv: 1711.02862] [INSPIRE]. 
[14] E.J. Chun et al., Probing leptogenesis, Int. J. Mod. Phys. A 33 (2018) 1842005 [arXiv: 1711.02865] [INSPIRE].

[15] C. Hagedorn, R.N. Mohapatra, E. Molinaro, C.C. Nishi and S.T. Petcov, CP violation in the lepton sector and implications for leptogenesis, Int. J. Mod. Phys. A 33 (2018) 1842006 [arXiv: 1711.02866] [INSPIRE].

[16] P.S.B. Dev, P. Di Bari, B. Garbrecht, S. Lavignac, P. Millington and D. Teresi, Flavor effects in leptogenesis, Int. J. Mod. Phys. A 33 (2018) 1842001 [arXiv:1711.02861] [inSPIRE].

[17] B. Dev, M. Garny, J. Klaric, P. Millington and D. Teresi, Resonant enhancement in leptogenesis, Int. J. Mod. Phys. A 33 (2018) 1842003 [arXiv:1711.02863] [INSPIRE].

[18] A. Anisimov, W. Buchmüller, M. Drewes and S. Mendizabal, Quantum leptogenesis I, Annals Phys. 326 (2011) 1998 [Erratum ibid. 338 (2013) 376] [arXiv: 1012.5821] [INSPIRE].

[19] A. Anisimov, W. Buchmüller, M. Drewes and S. Mendizabal, Leptogenesis from quantum interference in a thermal bath, Phys. Rev. Lett. 104 (2010) 121102 [arXiv:1001.3856] [INSPIRE].

[20] A. Anisimov, D. Besak and D. Bödeker, Thermal production of relativistic Majorana neutrinos: strong enhancement by multiple soft scattering, JCAP 03 (2011) 042 [arXiv: 1012.3784] [INSPIRE].

[21] D. Bödeker and M. Wörmann, Non-relativistic leptogenesis, JCAP 02 (2014) 016 [arXiv: 1311.2593] [INSPIRE].

[22] D. Bödeker and M. Laine, Kubo relations and radiative corrections for lepton number washout, JCAP 05 (2014) 041 [arXiv:1403.2755] [INSPIRE].

[23] D. Bödeker and M. Sangel, Lepton asymmetry rate from quantum field theory: NLO in the hierarchical limit, JCAP 06 (2017) 052 [arXiv: 1702.02155] [INSPIRE].

[24] S. Biondini, N. Brambilla and A. Vairo, CP asymmetry in heavy Majorana neutrino decays at finite temperature: the hierarchical case, JHEP 09 (2016) 126 [arXiv:1608.01979] [INSPIRE].

[25] W. Buchmüller, P. Di Bari and M. Plümacher, Cosmic microwave background, matter-antimatter asymmetry and neutrino masses, Nucl. Phys. B 643 (2002) 367 [Erratum ibid. 793 (2008) 362] [hep-ph/0205349] [INSPIRE].

[26] W. Buchmüller and M. Plümacher, Spectator processes and baryogenesis, Phys. Lett. B 511 (2001) 74 [hep-ph/0104189] [INSPIRE].

[27] E. Nardi, Y. Nir, J. Racker and E. Roulet, On Higgs and sphaleron effects during the leptogenesis era, JHEP 01 (2006) 068 [hep-ph/0512052] [INSPIRE].

[28] B. Garbrecht and P. Schwaller, Spectator effects during leptogenesis in the strong washout regime, JCAP 10 (2014) 012 [arXiv:1404.2915] [INSPIRE].

[29] M.L. Bellac, Thermal field theory, Cambridge University Press, Cambridge, U.K. (2011).

[30] L. Covi, E. Roulet and F. Vissani, CP violating decays in leptogenesis scenarios, Phys. Lett. B 384 (1996) 169 [hep-ph/9605319] [INSPIRE].

[31] B. Bergerhoff and C. Wetterich, The strongly interacting electroweak phase transition, Nucl. Phys. B 440 (1995) 171 [hep-ph/9409295] [INSPIRE].

[32] O. Philipsen, M. Teper and H. Wittig, On the mass spectrum of the $\mathrm{SU}(2)$ Higgs model in $(2+1)$-dimensions, Nucl. Phys. B 469 (1996) 445 [hep-lat/9602006] [INSPIRE]. 
[33] O. Philipsen, M. Teper and H. Wittig, Scalar gauge dynamics in $(2+1)$-dimensions at small and large scalar couplings, Nucl. Phys. B 528 (1998) 379 [hep-lat/9709145] [INSPIRE].

[34] J.I. Kapusta and C. Gale, Finite-temperature field theory: principles and applications, Cambridge University Press, Cambridge, U.K. (2011) [INSPIRE].

[35] D. Besak and D. Bödeker, Hard thermal loops for soft or collinear external momenta, JHEP 05 (2010) 007 [arXiv: 1002.0022] [INSPIRE].

[36] P.B. Arnold, G.D. Moore and L.G. Yaffe, Photon and gluon emission in relativistic plasmas, JHEP 06 (2002) 030 [hep-ph/0204343] [INSPIRE].

[37] M.E. Carrington, The effective potential at finite temperature in the Standard Model, Phys. Rev. D 45 (1992) 2933 [INSPIRE].

[38] GSL project contributors, GSL - GNU Scientific Library, http://www.gnu.org/software/gsl/, GNU project, Free Software Foundation (FSF), U.S.A. (2010).

[39] B. Schäling, The Boost C++ libraries, XML Press, U.S.A. (2014).

[40] B. Schrempp and M. Wimmer, Top quark and Higgs boson masses: interplay between infrared and ultraviolet physics, Prog. Part. Nucl. Phys. 37 (1996) 1 [hep-ph/9606386] [INSPIRE].

[41] H. Arason et al., Renormalization group study of the Standard Model and its extensions. 1. The Standard model, Phys. Rev. D 46 (1992) 3945 [InSPIRE]. 